\title{
Global Action-Angle Variables for the Periodic Toda Lattice
}

\author{
Andreas Henrici and Thomas Kappeler \\ Institut für Mathematik, Universität Zürich, Winterthurerstrasse 190, \\ CH-8057 Zürich, Switzerland
}

Correspondence to be sent to: thomas.kappeler@math.uzh.ch

In this paper, we construct global action-angle variables for the periodic Toda lattice.

\section{Introduction}

Consider the Toda lattice with period $N(N \geq 2)$,

$$
\dot{q}_{n}=\partial_{p_{n}} H, \quad \dot{p}_{n}=-\partial_{q_{n}} H
$$

for $n \in \mathbb{Z}$, where the (real) coordinates $\left(q_{n}, p_{n}\right)_{n \in \mathbb{Z}}$ satisfy $\left(q_{n+N}, p_{n+N}\right)=\left(q_{n}, p_{n}\right)$ for any $n \in \mathbb{Z}$ and the Hamiltonian $H_{\text {Toda }}$ is given by

$$
H_{\text {Toda }}=\frac{1}{2} \sum_{n=1}^{N} p_{n}^{2}+\alpha^{2} \sum_{n=1}^{N} e^{q_{n}-q_{n+1}}
$$

where $\alpha$ is a positive parameter, $\alpha>0$. For the standard Toda lattice, $\alpha=1$. The Toda lattice was introduced by Toda [18] and studied extensively in the sequel. It is an important model for an integrable system of $N$ particles in one space dimension with nearest neighbor interaction and belongs to the family of lattices introduced and numerically investigated by Fermi, Pasta, and Ulam in their seminal paper [3]. To prove the integrability

Received February 27, 2008; Revised February 27, 2008; Accepted March 10, 2008

Communicated by Prof. Percy Deift

(C) The Author 2008. Published by Oxford University Press. All rights reserved. For permissions,

please e-mail: journals.permissions@oxfordjournals.org. 
of the Toda lattice, Flaschka introduced in [4] the (noncanonical) coordinates

$$
b_{n}:=-p_{n} \in \mathbb{R}, \quad a_{n}:=\alpha e^{\frac{1}{2}\left(q_{n}-q_{n+1}\right)} \in \mathbb{R}_{>0} \quad(n \in \mathbb{Z}) .
$$

These coordinates describe the motion of the Toda lattice relative to the center of mass. Note that the total momentum is conserved by the Toda flow, hence any trajectory of the center of mass is a straight line.

In these coordinates, the Hamiltonian $H_{T o d a}$ takes the simple form

$$
H=\frac{1}{2} \sum_{n=1}^{N} b_{n}^{2}+\sum_{n=1}^{N} a_{n}^{2}
$$

and the equations of motion are

$$
\left\{\begin{array}{l}
\dot{b}_{n}=a_{n}^{2}-a_{n-1}^{2} \\
\dot{a}_{n}=\frac{1}{2} a_{n}\left(b_{n+1}-b_{n}\right)
\end{array} \quad(n \in \mathbb{Z}) .\right.
$$

Note that $\left(b_{n+N}, a_{n+N}\right)=\left(b_{n}, a_{n}\right)$ for any $n \in \mathbb{Z}$, and $\prod_{n=1}^{N} a_{n}=\alpha^{N}$. Hence we can identify the sequences $\left(b_{n}\right)_{n \in \mathbb{Z}}$ and $\left(a_{n}\right)_{n \in \mathbb{Z}}$ with the vectors $\left(b_{n}\right)_{1 \leq n \leq N} \in \mathbb{R}^{N}$ and $\left(a_{n}\right)_{1 \leq n \leq N} \in \mathbb{R}_{>0}^{N}$. Our aim is to study the normal form of the system of equations (2) on the phase space

$$
\mathcal{M}:=\mathbb{R}^{N} \times \mathbb{R}_{>0}^{N}
$$

This system is Hamiltonian with respect to the nonstandard Poisson structure $J \equiv J_{b, a}$, defined at a point $(b, a)=\left(\left(b_{n}, a_{n}\right)_{1 \leq n \leq N}\right.$ by

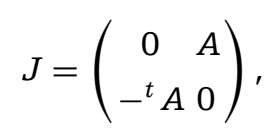

where $A$ is the $b$-independent $N \times N$-matrix

$$
A=\frac{1}{2}\left(\begin{array}{ccccc}
a_{1} & 0 & \ldots & 0 & -a_{N} \\
-a_{1} & a_{2} & 0 & \ddots & 0 \\
0 & -a_{2} & a_{3} & \ddots & \vdots \\
\vdots & \ddots & \ddots & \ddots & 0 \\
0 & \ldots & 0 & -a_{N-1} & a_{N}
\end{array}\right) .
$$


The Poisson bracket corresponding to equation (3) is then given by

$$
\begin{aligned}
\{F, G\}_{J}(b, a) & =\left\langle\left(\nabla_{b} F, \nabla_{a} F\right), J\left(\nabla_{b} G, \nabla_{a} G\right)\right\rangle_{\mathbb{R}^{2 N}} \\
& =\left\langle\nabla_{b} F, A \nabla_{a} G\right\rangle_{\mathbb{R}^{N}}-\left\langle\nabla_{a} F, A^{t} \nabla_{b} G\right\rangle_{\mathbb{R}^{N}},
\end{aligned}
$$

where $F, G \in C^{1}(\mathcal{N})$ and where $\nabla_{b}$ and $\nabla_{a}$ denote the gradients with respect to the $N$ vectors $b=\left(b_{1}, \ldots, b_{N}\right)$ and $a=\left(a_{1}, \ldots, a_{N}\right)$, respectively. Therefore, equations (2) can alternatively be written as $\dot{b}_{n}=\left\{b_{n}, H\right\}_{J}, \dot{a}_{n}=\left\{a_{n}, H\right\}_{J}(1 \leq n \leq N)$. Further note that

$$
\left\{b_{n}, a_{n}\right\}_{J}=\frac{a_{n}}{2} ; \quad\left\{b_{n+1}, a_{n}\right\}_{J}=-\frac{a_{n}}{2},
$$

while $\left\{b_{n}, a_{k}\right\}_{J}=0$ for any $n, k$ with $n \notin\{k, k+1\}$.

Since the matrix $A$ defined by equation (4) has rank $N-1$, the Poisson structure $J$ is degenerate. It admits the two Casimir functions

$$
C_{1}:=-\frac{1}{N} \sum_{n=1}^{N} b_{n} \quad \text { and } \quad C_{2}:=\left(\prod_{n=1}^{N} a_{n}\right)^{\frac{1}{N}}
$$

whose gradients $\nabla_{b, a} C_{i}=\left(\nabla_{b} C_{i}, \nabla_{a} C_{i}\right)(i=1,2)$, given by

$$
\begin{aligned}
& \nabla_{b} C_{1}=-\frac{1}{N}(1, \ldots, 1), \quad \nabla_{a} C_{1}=0, \\
& \nabla_{b} C_{2}=0, \quad \nabla_{a} C_{2}=\frac{C_{2}}{N}\left(\frac{1}{a_{1}}, \ldots, \frac{1}{a_{N}}\right),
\end{aligned}
$$

are linearly independent at each point $(b, a)$ of $\mathcal{M}$. (A smooth function $C: \mathcal{M} \rightarrow \mathbb{R}$ is a Casimir function for $J$ if $\{C, \cdot\}_{J} \equiv 0$.)

The main result of this paper is the following one.

Theorem 1.1. The periodic Toda lattice admits globally defined action-angle variables. More precisely,

(i) There exist real analytic functions $\left(I_{n}\right)_{1 \leq n \leq N-1}$ on $\mathcal{M}$ which are pairwise in involution and which Poisson commute with the Toda Hamiltonian $H$ and the two Casimir functions $C_{1}, C_{2}$, i.e. for any $1 \leq m, n \leq N-1, i=1,2$,

$$
\left\{I_{m}, I_{n}\right\}_{J}=0 \quad \text { on } \mathcal{M}
$$

and

$$
\left\{H, I_{n}\right\}_{J}=0 \quad \text { and } \quad\left\{C_{i}, I_{n}\right\}_{J}=0 \quad \text { on } \mathcal{M} \text {. }
$$


(ii) For any $1 \leq n \leq N-1$, there exist a real analytic submanifold $D_{n}$ of codimension 2 and a function $\theta_{n}: \mathcal{M} \backslash D_{n} \rightarrow \mathbb{R}$, defined $\bmod 2 \pi$ and real analytic when considered $\bmod \pi$, so that on $\mathcal{M} \backslash \bigcup_{n=1}^{N-1} D_{n},\left(\theta_{n}\right)_{1 \leq n \leq N-1}$ and $\left(I_{n}\right)_{1 \leq n \leq N-1}$ are conjugate variables. More precisely, for any $1 \leq m, n \leq N-1, i=1,2$

$$
\left\{I_{m}, \theta_{n}\right\}_{J}=\delta_{m n} \quad \text { and } \quad\left\{C_{i}, \theta_{n}\right\}_{J}=0 \quad \text { on } \mathcal{M} \backslash D_{n}
$$

and

$$
\left\{\theta_{m}, \theta_{n}\right\}_{J}=0 \quad \text { on } \mathcal{M} \backslash\left(D_{m} \cup D_{n}\right) .
$$

Let $\mathcal{M}_{\beta, \alpha}:=\left\{(b, a) \in \mathbb{R}^{2 N}:\left(C_{1}, C_{2}\right)=(\beta, \alpha)\right\}$ denote the level set of $\left(C_{1}, C_{2}\right)$ for $(\beta, \alpha) \in$ $\mathbb{R} \times \mathbb{R}_{>0}$. Note that $\left(-\beta 1_{N}, \alpha 1_{N}\right) \in \mathcal{M}_{\beta, \alpha}$, where $1_{N}=(1, \ldots, 1) \in \mathbb{R}^{N}$. As the gradients $\nabla_{b, a} C_{1}$ and $\nabla_{b, a} C_{2}$ are linearly independent everywhere on $\mathcal{M}$, the sets $\mathcal{M}_{\beta, \alpha}$ are (real analytic) submanifolds of $\mathcal{M}$ of codimension 2. Furthermore, the Poisson structure $J$, restricted to $\mathcal{M}_{\beta, \alpha}$, becomes nondegenerate everywhere on $\mathcal{M}_{\beta, \alpha}$ and therefore induces a symplectic structure $v_{\beta, \alpha}$ on $\mathcal{M}_{\beta, \alpha}$. In this way, we obtain a symplectic foliation of $\mathcal{M}$ with $\mathcal{M}_{\beta, \alpha}$ being the symplectic leaves.

Corollary 1.2. On each symplectic leaf $\mathcal{M}_{\beta, \alpha}$, the action variables $\left(I_{n}\right)_{1 \leq n \leq N-1}$ are a maximal set of functionally independent integrals in involution of the periodic Toda lattice.

In subsequent work [9], we will use Theorem 1.1 to construct global Birkhoff coordinates for the periodic Toda lattice. More precisely, we introduce the model space $\mathcal{P}:=\mathbb{R}^{2(N-1)} \times \mathbb{R} \times \mathbb{R}_{>0}$ endowed with the degenerate Poisson structure $J_{0}$ whose symplectic leaves are $\mathbb{R}^{2(N-1)} \times\{\beta\} \times\{\alpha\}$, endowed with the standard Poisson structure, and prove the following theorem.

Theorem 1.3. There exists a real analytic, canonical diffeomorphism

$$
\begin{aligned}
\Omega:(\mathcal{M}, J) & \rightarrow\left(\mathcal{P}, J_{0}\right) \\
(b, a) & \mapsto\left(\left(x_{n}, y_{n}\right)_{1 \leq n \leq N-1}, C_{1}, C_{2}\right)
\end{aligned}
$$

such that the coordinates $\left(x_{n}, y_{n}\right)_{1 \leq n \leq N-1}, C_{1}, C_{2}$ are global Birkhoff coordinates for the periodic Toda lattice, i.e. $\left(x_{n}, y_{n}\right)_{1 \leq n \leq N-1}$ are canonical coordinates, $C_{1}, C_{2}$ are the Casimirs, and the transformed Toda Hamiltonian $\hat{H}=H \circ \Omega^{-1}$ is a function of the actions $\left(I_{n}\right)_{1 \leq n \leq N-1}$ and $C_{1}, C_{2}$ alone. 
In [10], we used Theorem 1.3 to obtain a KAM theorem for Hamiltonian perturbations of the periodic Toda lattice.

Related work. Theorem 1.1 and Theorem 1.3 improve on earlier work on the normal form of the periodic Toda lattice in [1, 2]. In particular, we construct global Birkhoff coordinates on all of $\mathcal{M}$ instead of a single symplectic leaf and show that techniques recently developed for treating the $\mathrm{KdV}$ equation (cf. [11, 12]) and the defocusing NLS equation (cf. $[8,15])$ can also be applied for the Toda lattice.

Outline of the paper. In Section 2, we review the Lax pair of the periodic Toda lattice and collect some auxiliary results on the spectrum of the Jacobi matrix $L(b, a)$ associated to an element $(b, a) \in \mathcal{M}$. In Section 3, we study the action variables $\left(I_{n}\right)_{1 \leq n \leq N-1}$, and in Section 4 we define the angle variables $\left(\theta_{n}\right)_{1 \leq n \leq N-1}$ on $\mathcal{M} \backslash \cup_{n=1}^{n} D_{n}$ using holomorphic differentials defined on the hyperelliptic Riemann surface associated to the spectrum of $L(b, a)$. In Sections 5 and 6, we establish formulas of the gradients of the actions and angles in terms of products of fundamental solutions and prove orthogonality relations between such products which are then used in Section 7 to show that $\left(I_{n}\right)_{1 \leq n \leq N-1}$ and $\left(\theta_{n}\right)_{1 \leq n \leq N-1}$ are canonical variables and to prove Theorem 1.1 and Corollary 1.2.

\section{Preliminaries}

It is well known (cf. [18]) that the system (2) admits a Lax pair formulation $\dot{L}=\frac{\partial L}{\partial t}=[B, L]$, where $L \equiv L^{+}(b, a)$ is the periodic Jacobi matrix defined by

$$
L^{ \pm}(b, a):=\left(\begin{array}{ccccc}
b_{1} & a_{1} & 0 & \ldots & \pm a_{N} \\
a_{1} & b_{2} & a_{2} & \ddots & \vdots \\
0 & a_{2} & b_{3} & \ddots & 0 \\
\vdots & \ddots & \ddots & \ddots & a_{N-1} \\
\pm a_{N} & \ldots & 0 & a_{N-1} & b_{N}
\end{array}\right),
$$

and $B$ the skew-symmetric matrix

$$
B=\left(\begin{array}{ccccc}
0 & a_{1} & 0 & \ldots & -a_{N} \\
-a_{1} & 0 & a_{2} & \ddots & \vdots \\
0 & -a_{2} & \ddots & \ddots & 0 \\
\vdots & \ddots & \ddots & \ddots & a_{N-1} \\
a_{N} & \ldots & 0 & -a_{N-1} & 0
\end{array}\right) .
$$

Hence the flow of $\dot{L}=[B, L]$ is isospectral. 
Proposition 2.1. For a solution $(b(t), a(t))$ of the periodic Toda lattice (2), the eigenvalues $\left(\lambda_{j}^{+}\right)_{1 \leq j \leq N}$ of $L(b(t), a(t))$ are conserved quantities.

Let us now collect a few results from [16] and [18] of the spectral theory of Jacobi matrices needed in the sequel. Denote by $\mathcal{M}^{\mathbb{C}}$ the complexification of the phase space $\mathcal{M}$,

$$
\mathcal{M}^{\mathbb{C}}=\left\{(b, a) \in \mathbb{C}^{2 N}: \operatorname{Re} a_{j}>0 \quad \forall 1 \leq j \leq N\right\}
$$

For $(b, a) \in \mathcal{M}^{\mathbb{C}}$, we consider for any complex number $\lambda$ the difference equation

$$
\left(R_{b, a} y\right)(k)=\lambda y(k) \quad(k \in \mathbb{Z})
$$

where $y(\cdot)=y(k)_{k \in \mathbb{Z}} \in \mathbb{C}^{\mathbb{Z}}$ and $R_{b, a}$ is the difference operator

$$
R_{b, a}=a_{k-1} S^{-1}+b_{k} S^{0}+a_{k} S^{1}
$$

with $S^{m}$ denoting the shift operator of order $m \in \mathbb{Z}$, that is,

$$
\left(S^{m} y\right)(k)=y(k+m) \quad \text { for } \quad k \in \mathbb{Z} .
$$

Fundamental solutions. The two fundamental solutions $y_{1}(\cdot, \lambda)$ and $y_{2}(\cdot, \lambda)$ of equation (11) are defined by the standard initial conditions $y_{1}(0, \lambda)=1, y_{1}(1, \lambda)=0$, and $y_{2}(0, \lambda)=0, y_{2}(1, \lambda)=1$. They satisfy the Wronskian identity

$$
W(n):=y_{1}(n, \lambda) y_{2}(n+1, \lambda)-y_{1}(n+1, \lambda) y_{2}(n, \lambda)=\frac{a_{N}}{a_{n}} .
$$

Note that for $n=N$ one gets

$$
W(N)=1
$$

For each $k \in \mathbb{N}, y_{i}(k, \lambda, b, a), i=1,2$, is a polynomial in $\lambda$ of degree at most $k-1$ and depends real analytically on $(b, a)$ (see [16]). In particular, one easily verifies that $y_{2}(N+$ $1, \lambda, b, a)$ is a polynomial in $\lambda$ of degree $N$ with leading coefficient $\alpha^{-N}$.

Wronskian. More generally, one defines for any two sequences $(v(n))_{n \in \mathbb{Z}}$ and $(w(n))_{n \in \mathbb{Z}}$ the Wronskian sequence $(W(n))_{n \in \mathbb{Z}}=(W(v, w)(n))_{n \in \mathbb{Z}}$ by

$$
W(n):=v(n) w(n+1)-v(n+1) w(n) .
$$

Let us recall the following properties of the Wronskian, which can be easily verified. 


\section{Lemma 2.2.}

(i) If $y$ and $z$ are solutions of equation (11) for $\lambda=\lambda_{1}$ and $\lambda=\lambda_{2}$, respectively, then $W=W(y, z)$ satisfies for any $k \in \mathbb{Z}$

$$
a_{k} W(k)=a_{k-1} W(k-1)+\left(\lambda_{2}-\lambda_{1}\right) y(k) z(k) .
$$

(ii) If $y(\cdot, \lambda)$ is a one-parameter family of solutions of equation (11) which is continuously differentiable with respect to the parameter $\lambda$ and $\dot{y}(k, \lambda):=$ $\frac{\partial}{\partial \lambda} y(k, \lambda)$, then $W=W(y, \dot{y})$ satisfies for any $k \in \mathbb{Z}$

$$
a_{k} W(k)=a_{k-1} W(k-1)+y(k, \lambda)^{2} .
$$

Discriminant. We denote by $\Delta(\lambda) \equiv \Delta(\lambda, b, a)$ the discriminant of equation (11), defined by

$$
\Delta(\lambda):=y_{1}(N, \lambda)+y_{2}(N+1, \lambda) .
$$

In the sequel, we will often write $\Delta_{\lambda}$ for $\Delta(\lambda)$. Note that $y_{2}(N+1, \lambda)$ is a polynomial in $\lambda$ of degree $N$ with leading term $\alpha^{-N} \lambda^{N}$, whereas $y_{1}(N, \lambda)$ is a polynomial in $\lambda$ of degree less than $N$, hence $\Delta(\lambda, b, a)$ is a polynomial in $\lambda$ of degree $N$ with leading term $\alpha^{-N} \lambda^{N}$, and it depends real analytically on $(b, a)$ (see e.g. [18]). According to Floquet's Theorem (see e.g. [17]), for $\lambda \in \mathbb{C}$ given, equation (11) admits a periodic or antiperiodic solution of period $N$ if the discriminant $\Delta(\lambda)$ satisfies $\Delta(\lambda)=+2$ or $\Delta(\lambda)=-2$, respectively. (These solutions correspond to eigenvectors of $L^{+}$or $L^{-}$, respectively, with $L^{ \pm}$defined by equation (10).) It turns out to be more convenient to combine these two cases by considering the periodic Jacobi matrix $Q \equiv Q(b, a)$ of size $2 N$ defined by

$$
Q=\left(\begin{array}{cccc|cccc}
b_{1} & a_{1} & \ldots & 0 & 0 & \ldots & 0 & a_{N} \\
a_{1} & b_{2} & \ddots & \vdots & 0 & \ldots & & 0 \\
\vdots & \ddots & \ddots & a_{N-1} & \vdots & & & \vdots \\
0 & \ddots & a_{N-1} & b_{N} & a_{N} & \ldots & 0 & 0 \\
\hline 0 & \ldots & 0 & a_{N} & b_{1} & a_{1} & \ldots & 0 \\
0 & \ldots & & 0 & a_{1} & b_{2} & \ddots & \vdots \\
\vdots & & & \vdots & \vdots & \ddots & \ddots & a_{N-1} \\
a_{N} & \ldots & 0 & 0 & 0 & \ddots & a_{N-1} & b_{N}
\end{array}\right) .
$$




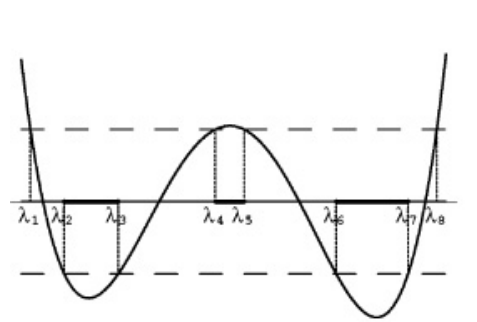

(a) $N=4$

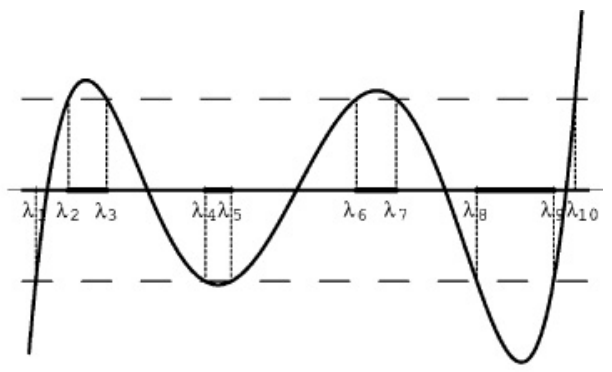

(b) $N=5$

Fig. 1. Examples of the discriminant $\Delta(\lambda)$.

Then the spectrum of the matrix $Q$ is the union of the spectra of the matrices $L^{+}$and $L^{-}$ and therefore the zero set of the polynomial $\Delta_{\lambda}^{2}-4$. The function $\Delta_{\lambda}^{2}-4$ is a polynomial in $\lambda$ of degree $2 N$ and admits a product representation

$$
\Delta_{\lambda}^{2}-4=\alpha^{-2 N} \prod_{j=1}^{2 N}\left(\lambda-\lambda_{j}\right)
$$

The factor $\alpha^{-2 N}$ in equation (18) comes from the above-mentioned fact that the leading term of $\Delta(\lambda)$ is $\alpha^{-N} \lambda^{N}$.

For any $(b, a) \in \mathcal{M}$, the matrix $Q$ is symmetric and hence the eigenvalues $\left(\lambda_{j}\right)_{1 \leq j \leq 2 N}$ of $Q$ are real. When listed in increasing order and with their algebraic multiplicities, they satisfy the following relations (cf. [16]):

$$
\lambda_{1}<\lambda_{2} \leq \lambda_{3}<\lambda_{4} \leq \lambda_{5}<\cdots \lambda_{2 N-2} \leq \lambda_{2 N-1}<\lambda_{2 N}
$$

As explained above, the $\lambda_{j}$ are periodic or antiperiodic eigenvalues of $L$ and thus eigenvalues of $L^{+}$or $L^{-}$according to whether $\Delta(\lambda)=2$ or $\Delta(\lambda)=-2$. One has (cf. [16])

$$
\Delta\left(\lambda_{1}\right)=(-1)^{N} \cdot 2, \quad \Delta\left(\lambda_{2 n}\right)=\Delta\left(\lambda_{2 n+1}\right)=(-1)^{n+N} \cdot 2, \quad \Delta\left(\lambda_{2 N}\right)=2 .
$$

See Fig. 1 for an illustration in the cases $N=4$ and $N=5$.

Since $\Delta_{\lambda}$ is a polynomial of degree $N, \dot{\Delta}_{\lambda} \equiv \dot{\Delta}(\lambda)=\frac{d}{d \lambda} \Delta(\lambda)$ is a polynomial of degree $N-1$, and it admits a product representation of the form

$$
\dot{\Delta}_{\lambda}=N \alpha^{-N} \prod_{k=1}^{N-1}\left(\lambda-\dot{\lambda}_{k}\right) .
$$


The zeroes $\left(\dot{\lambda}_{n}\right)_{1 \leq n \leq N-1}$ of $\dot{\Delta}_{\lambda}$ satisfy $\lambda_{2 n} \leq \dot{\lambda}_{n} \leq \lambda_{2 n+1}$ for any $1 \leq n \leq N-1$. The intervals $\left(\lambda_{2 n}, \lambda_{2 n+1}\right)$ are referred to as the $n$th spectral gap and $\gamma_{n}:=\lambda_{2 n+1}-\lambda_{2 n}$ as the $n t h$ gap length. Note that $|\Delta(\lambda)|>2$ on the spectral gaps. We say that the $n$th gap is open if $\gamma_{n}>0$ and collapsed otherwise. The set of elements $(b, a) \in \mathcal{M}$ for which the $n$th gap is collapsed is denoted by $D_{n}$,

$$
D_{n}:=\left\{(b, a) \in \mathcal{M}: \gamma_{n}=0\right\}
$$

By writing the condition $\gamma_{n}=0$ as $\gamma_{n}^{2}=0$ and exploiting the fact that $\gamma_{n}^{2}$ (unlike $\gamma_{n}$ ) is a real analytic function on $\mathcal{M}$, it can be shown as in [12] that $D_{n}$ is a real analytic submanifold of $\mathcal{M}$ of codimension 2.

Isolating neighborhoods. Let $(b, a) \in \mathcal{M}$ be given. The strict inequality $\lambda_{2 n-1}<\lambda_{2 n}$ guarantees the existence of a family of mutually disjoint open subsets $\left(U_{n}\right)_{1 \leq n \leq N-1}$ of $\mathbb{C}$, so that for any $1 \leq n \leq N-1, U_{n}$ is a neighborhood of the closed interval $\left[\lambda_{2 n}, \lambda_{2 n+1}\right]$. Such a family of neighborhoods is referred to as a family of isolating neighborhoods (for $(b, a))$.

In the case where $(b, a) \in \mathcal{M}^{\mathbb{C}}$, we list the eigenvalues $\left(\lambda_{j}\right)_{1 \leq j \leq 2 N}$ in lexicographic ordering $\lambda_{1} \prec \lambda_{2} \prec \lambda_{3} \prec \cdots \prec \lambda_{2 N}$.

The lexicographic ordering $a \prec b$ for complex numbers $a$ and $b$ is defined by

$$
a \prec b \quad \Longleftrightarrow \quad\left\{\begin{array}{l}
\operatorname{Re} a<\operatorname{Re} b \\
\text { or } \\
\operatorname{Re} a=\operatorname{Re} b \text { and } \operatorname{Im} a \leq \operatorname{Im} b .
\end{array}\right.
$$

We then extend the gap lengths $\gamma_{n}$ to all of $\mathcal{M}^{\mathbb{C}}$ by

$$
\gamma_{n}:=\lambda_{2 n+1}-\lambda_{2 n} \quad(1 \leq n \leq N-1)
$$

and define

$$
D_{n}^{\mathbb{C}}:=\left\{(b, a) \in \mathcal{W}: \gamma_{n}=0\right\}
$$

In the sequel, we will omit the superscript and always write $D_{n}$ for $D_{n}^{\mathbb{C}}$.

Similarly, we do this for the zeroes $\left(\dot{\lambda}_{n}\right)_{1 \leq n \leq N-1}$ of $\dot{\Delta}_{\lambda}$. The $\lambda_{i}$ 's and $\dot{\lambda}_{i}$ 's no longer depend continuously on $(b, a) \in \mathcal{M}^{\mathbb{C}}$. However, if we choose a small enough complex neighborhood $\mathcal{W}$ of $\mathcal{M}$ in $\mathcal{M}^{\mathbb{C}}$, then for any $(b, a) \in \mathcal{W}$, the closed intervals $G_{n} \subseteq \mathbb{C}(1 \leq n \leq N-1)$ 
defined by

$$
G_{n}:=\left\{(1-t) \lambda_{2 n}+t \lambda_{2 n+1}: 0 \leq t \leq 1\right\}
$$

are pairwise disjoint, and hence, as in the real case, there exists a family of isolating neighborhoods $\left(U_{n}\right)_{1 \leq n \leq N-1}$.

Lemma 2.3. There exists a neighborhood $\mathcal{W}$ of $\mathcal{M}$ in $\mathcal{M}^{\mathbb{C}}$ such that for any $(b, a) \in \mathcal{W}$, there are neighborhoods $U_{n}$ of $G_{n}$ in $\mathbb{C}(1 \leq n \leq N-1)$ which are pairwise disjoint.

Remark 2.4. In the sequel, we will have to shrink the complex neighborhood $\mathcal{W}$ several times, but continue to denote it by the same letter.

Contours $\Gamma_{n}$. For any $(b, a) \in \mathcal{W}$ and any $1 \leq n \leq N-1$, we denote by $\Gamma_{n}$ a circuit in $U_{n}$ around $G_{n}$ with counterclockwise orientation.

Isospectral set. For $(b, a) \in \mathcal{M}$, the set Iso $(b, a)$ of all elements $\left(b^{\prime}, a^{\prime}\right) \in \mathcal{M}$ so that $Q\left(b^{\prime}, a^{\prime}\right)$ has the same spectrum as $Q(b, a)$ is described with the help of the Dirichlet eigenvalues $\mu_{1}<\mu_{2}<\cdots<\mu_{N-1}$ of equation (11) defined by

$$
y_{1}\left(N+1, \mu_{n}\right)=0 .
$$

They coincide with the eigenvalues of the $(N-1) \times(N-1)$-matrix $L_{2}=L_{2}(b, a)$ given by

$$
\left(\begin{array}{ccccc}
b_{2} & a_{2} & 0 & \ldots & 0 \\
a_{2} & \ddots & \ddots & \ddots & \vdots \\
0 & \ddots & \ddots & \ddots & 0 \\
\vdots & \ddots & \ddots & \ddots & a_{N-1} \\
0 & \ldots & 0 & a_{N-1} & b_{N}
\end{array}\right)
$$

In the sequel, we will also refer to $\mu_{1}, \ldots, \mu_{N-1}$ as the Dirichlet eigenvalues of $L(b, a)$. Evaluating the Wronskian identity (13) at $\lambda=\mu_{n}$ one sees that $\mu_{n}$ lies in the closure of the $n$th spectral gap. More precisely, substituting $y_{1}\left(N+1, \mu_{n}\right)=0$ in identity (13) with $\lambda=\mu_{n}$ yields

$$
y_{1}\left(N, \mu_{n}\right)_{Y_{2}}\left(N+1, \mu_{n}\right)=1 .
$$


Hence the value of the discriminant at $\mu_{n}$ is given by

$$
\Delta\left(\mu_{n}\right)=y_{2}\left(N+1, \mu_{n}\right)+\frac{1}{y_{2}\left(N+1, \mu_{n}\right)}
$$

and $\left|\Delta\left(\mu_{n}\right)\right| \geq 2$. By Lemma 2.6, given the point $(b, a)$ with $b_{1}=\ldots=b_{N}=\beta$ and $a_{1}=\ldots=$ $a_{N}=\alpha$, one has $\lambda_{2 n}=\lambda_{2 n+1}$ and hence $\mu_{n}=\lambda_{2 n}$ for any $1 \leq n \leq N-1$. It then follows from a straightforward deformation argument that $\lambda_{2 n} \leq \mu_{n} \leq \lambda_{2 n+1}$ everywhere in the real space $\mathcal{M}$.

Conversely, according to van Moerbeke [16], given any (real) Jacobi matrix $Q$ with spectrum $\lambda_{1}<\lambda_{2} \leq \lambda_{3}<\lambda_{4} \leq \lambda_{5}<\cdots \lambda_{2 N-2} \leq \lambda_{2 N-1}<\lambda_{2 N}$ and any sequence $\left(\mu_{n}\right)_{1 \leq n \leq N-1}$ with $\lambda_{2 n} \leq \mu_{n} \leq \lambda_{2 n+1}$ for $n=1, \ldots, N-1$, there are exactly $2^{r} N$-periodic Jacobi matrices $Q$ with spectrum $\left(\lambda_{n}\right)_{1 \leq n \leq 2 N}$ and Dirichlet spectrum $\left(\mu_{n}\right)_{1 \leq n \leq N-1}$, where $r$ is the number of $n$ 's with $\lambda_{2 n}<\mu_{n}<\lambda_{2 n+1}$.

In the case where $(b, a) \in \mathcal{M}^{\mathbb{C}}$, we continue to define the Dirichlet eigenvalues $\left(\mu_{n}\right)_{1 \leq n \leq N-1}$ by equation (25), and we list them in lexicographic ordering $\mu_{1} \prec \mu_{2} \prec \cdots \prec$ $\mu_{N-1}$. Then the $\mu_{i}$ 's no longer depend continuously on $(b, a) \in \mathcal{M}^{\mathbb{C}}$. However, if we choose the complex neighborhood $\mathcal{W}$ of $\mathcal{N}$ in $\mathcal{M}^{\mathbb{C}}$ of Lemma 2.3 small enough, then for any $(b, a) \in \mathcal{W}$ and $1 \leq n \leq N-1, \mu_{n}$ is contained in the neighborhood $U_{n}$ of $G_{n}$ (but not necessarily in $G_{n}$ itself).

Riemann surface $\Sigma_{b, a}$. Denote by $\Sigma_{b, a}$ the Riemann surface obtained as the compactification of the affine curve $\mathcal{C}_{b, a}$ defined by

$$
\left\{(\lambda, z) \in \mathbb{C}^{2}: z^{2}=\Delta^{2}(\lambda, b, a)-4\right\}
$$

Note that $\mathcal{C}_{b, a}$ and $\Sigma_{b, a}$ are spectral invariants. (Strictly speaking, $\Sigma_{b, a}$ is a Riemann surface only if the spectrum of $Q(b, a)$ is simple-see e.g. Appendix A in [17] for details in this case. If the spectrum of $Q(b, a)$ is not simple, $\Sigma(b, a)$ becomes a Riemann surface after doubling the multiple eigenvalues-see e.g. Section 2 of [13].)

Dirichlet divisors. To the Dirichlet eigenvalue $\mu_{n}(1 \leq n \leq N-1)$ we associate the point $\mu_{n}^{*}$ on the surface $\Sigma_{b, a}$ ，

$$
\mu_{n}^{*}:=\left(\mu_{n}, \sqrt[*]{\Delta_{\mu_{n}}^{2}-4}\right) \text { with } \sqrt[*]{\Delta_{\mu_{n}}^{2}-4}:=y_{1}\left(N, \mu_{n}\right)-y_{2}\left(N+1, \mu_{n}\right),
$$

where we used that, in view of equation (26) and the Wronskian identiy 14,

$$
\Delta_{\mu_{n}}^{2}-4=\left(y_{1}\left(N, \mu_{n}\right)-y_{2}\left(N+1, \mu_{n}\right)\right)^{2} .
$$


Standard root. The standard root or $s$-root for short, $\sqrt[s]{1-\lambda^{2}}$, is defined for $\lambda \in \mathbb{C} \backslash[-1,1]$ by

$$
\sqrt[s]{1-\lambda^{2}}:=i \lambda \sqrt[+]{1-\lambda^{-2}}
$$

More generally, we define for $\lambda \in \mathbb{C} \backslash\{t a+(1-t) b \mid 0 \leq t \leq 1\}$ the $s$-root of a radicand of the form $(b-\lambda)(\lambda-a)$ with $a \prec b, a \neq b$ by

$$
\sqrt[s]{(b-\lambda)(\lambda-a)}:=\frac{\gamma}{2} \sqrt[s]{1-w^{2}}
$$

where $\gamma:=b-a, \tau:=\frac{b+a}{2}$, and $w:=\frac{\lambda-\tau}{\gamma / 2}$.

Canonical sheet and canonical root. For $(b, a) \in \mathcal{M}$, the canonical sheet of $\Sigma_{b, a}$ is given by the set of points $\left(\lambda, \sqrt[c]{\Delta_{\lambda}^{2}-4}\right)$ in $\mathcal{C}_{b, a}$, where the $c$-root $\sqrt[c]{\Delta_{\lambda}^{2}-4}$ is defined on $\mathbb{C} \backslash \bigcup_{n=0}^{N}\left(\lambda_{2 n}, \lambda_{2 n+1}\right)$ (where $\lambda_{0}:=-\infty$ and $\lambda_{2 N+1}:=\infty$ ) and determined by the sign condition

$$
-i \sqrt[c]{\Delta_{\lambda}^{2}-4}>0 \text { for } \lambda_{2 N-1}<\lambda<\lambda_{2 N}
$$

As a consequence, one has for any $1 \leq n \leq N$

$$
\operatorname{sign} \sqrt[c]{\Delta_{\lambda-i 0}^{2}-4}=(-1)^{N+n-1} \text { for } \lambda_{2 n}<\lambda<\lambda_{2 n+1} .
$$

The definition of the canonical sheet and the $c$-root can be extended to the neighborhood $\mathcal{W}$ of $\mathcal{M}$ in $\mathcal{M}^{\mathbb{C}}$ of Lemma 2.3.

The $s$-root and the $c$-root will be used together in the following way: By the product representations (20) and (18) of $\dot{\Delta}_{\lambda}$ and $\Delta_{\lambda}^{2}-4$, respectively, one sees that for any $(b, a)$ in $\mathcal{W} \backslash D_{n}$ with $1 \leq n \leq N-1$,

$$
\frac{\dot{\Delta}_{\lambda}}{\sqrt[c]{\Delta_{\lambda}^{2}-4}}=\frac{N\left(\lambda-\dot{\lambda}_{n}\right)}{\sqrt[s]{\left(\lambda_{2 n+1}-\lambda\right)\left(\lambda-\lambda_{2 n}\right)}} \chi_{n}(\lambda) \quad \forall \lambda \in \Gamma_{n},
$$

where

$$
\chi_{n}(\lambda)=\frac{(-1)^{N+n-1}}{\sqrt[+]{\left(\lambda-\lambda_{1}\right)\left(\lambda_{2 N}-\lambda\right)}} \prod_{m \neq n} \frac{\lambda-\dot{\lambda}_{m}}{\sqrt[+]{\left(\lambda-\lambda_{2 m+1}\right)\left(\lambda-\lambda_{2 m}\right)}} .
$$


Note that the principal branches of the square roots in equation (35) are well defined for $\lambda$ near $G_{n}$ and that the function $\chi_{n}$ is analytic and nonvanishing on $U_{n}$. In addition, for $(b, a)$ real, $\chi_{n}$ is non-negative on the interval $\left(\lambda_{2 n}, \lambda_{2 n+1}\right)$.

Abelian differentials. Let $(b, a) \in \mathcal{M}$ and $1 \leq n \leq N-1$. Then there exists a unique polynomial $\psi_{n}(\lambda)$ of degree at most $N-2$ such that for any $1 \leq k \leq N-1$,

$$
\frac{1}{2 \pi} \int_{c_{k}} \frac{\psi_{n}(\lambda)}{\sqrt{\Delta_{\lambda}^{2}-4}} d \lambda=\delta_{k n} .
$$

Here, for any $1 \leq k \leq N-1, c_{k}$ denotes the lift of the contour $\Gamma_{k}$ to the canonical sheet of $\Sigma_{b, a}$. For any $k \neq n$ with $\lambda_{2 k} \neq \lambda_{2 k+1}$, it follows from equation (36) that

$$
\frac{1}{\pi} \int_{\lambda_{2 k}}^{\lambda_{2 k+1}} \frac{\psi_{n}(\lambda)}{\sqrt[+]{\Delta_{\lambda}^{2}-4}} d \lambda=0 .
$$

Hence in every gap $\left(\lambda_{2 k}, \lambda_{2 k+1}\right)$ with $k \neq n$, the polynomial $\psi_{n}$ has a zero which we denote by $\sigma_{k}^{n}$. If $\lambda_{2 k}=\lambda_{2 k+1}$, then it follows from equation (36) and Cauchy's theorem that $\sigma_{k}^{n}=$ $\lambda_{2 k}=\lambda_{2 k+1}$. As $\psi_{n}(\lambda)$ is a polynomial of degree at most $N-2$, one has

$$
\psi_{n}(\lambda)=M_{n} \prod_{\substack{1 \leq k \leq N-1 \\ k \neq n}}\left(\lambda-\sigma_{k}^{n}\right),
$$

where $M_{n} \equiv M_{n}(b, a) \neq 0$.

In a straightforward way, one can prove that there exists a neighborhood $\mathcal{W}$ of $\mathcal{M}$ in $\mathcal{M}_{\mathbb{C}}$, so that for any $(b, a) \in \mathcal{W}$ and any $1 \leq n \leq N-1$, there is a unique polynomial $\psi_{n}(\lambda)$ of degree at most $N-2$ satisfying equation (36) for any $1 \leq k \leq N-1$ as well as the product representation (38), and so that the zeroes are analytic functions on $\mathcal{W}$.

Lemma 2.5. Let $1 \leq n \leq N-1$ be fixed. Then the zeroes $\left(\dot{\lambda}_{k}\right)_{1 \leq k \leq N-1}$ of $\dot{\Delta}(\lambda)$ and $\left(\sigma_{k}^{n}\right)_{1 \leq k \leq N-1, k \neq n}$ of $\psi_{n}(\lambda)$ satisfy the estimates

$$
\begin{aligned}
\dot{\lambda}_{k}-\tau_{k} & =O\left(\gamma_{k}^{2}\right), \\
\sigma_{k}^{n}-\tau_{k} & =O\left(\gamma_{k}^{2}\right)
\end{aligned}
$$

near any given point $(b, a) \in \mathcal{W}$, where $\tau_{k}=\frac{1}{2}\left(\lambda_{2 k+1}+\lambda_{2 k}\right)$. 
Proof. To verify equation (39), write $\Delta_{\lambda}^{2}-4$ in the form

$$
\Delta_{\lambda}^{2}-4=\left(\lambda-\lambda_{2 n}\right)\left(\lambda_{2 n+1}-\lambda\right) p_{n}(\lambda)
$$

where $p_{n}$ is a polynomial which does not vanish for $\lambda \in U_{n}$. Then equation (39) follows by differentiating equation (41) with respect to $\lambda$ at $\dot{\lambda}_{n}$.

Fix $1 \leq k, n \leq N-1$ with $k \neq n$. In the first step, we prove that $\sigma_{k}^{n}-\tau_{k}=O\left(\gamma_{k}\right)$ near any given point $(b, a) \in \mathcal{W}$. If $\gamma_{k}=0$, then $\sigma_{k}^{n}=\tau_{k}$, and equation (40) is clearly fulfilled. Hence, we assume in the sequel that $\gamma_{k} \neq 0$. By the product formulas (38) and (18) for $\psi_{n}(\lambda)$ and $\Delta_{\lambda}^{2}-4$, respectively, we obtain, for $\lambda$ near $G_{k}$,

$$
\frac{\psi_{n}(\lambda)}{\sqrt[c]{\Delta_{\lambda}^{2}-4}}=\frac{\lambda-\sigma_{k}^{n}}{\sqrt[s]{\left(\lambda_{2 k+1}-\lambda\right)\left(\lambda-\lambda_{2 k}\right)}} \zeta_{k}^{n}(\lambda)
$$

where

$$
\zeta_{k}^{n}(\lambda)=\frac{M_{n}^{\prime}(b, a)}{\left(\lambda-\sigma_{n}^{n}\right) \sqrt[+]{\left(\lambda-\lambda_{1}\right)\left(\lambda_{2 N}-\lambda\right)}} \prod_{m \neq k} \frac{\lambda-\sigma_{m}^{n}}{\sqrt[+]{\left(\lambda_{2 m+1}-\lambda\right)\left(\lambda_{2 m}-\lambda\right)}}
$$

with $\sigma_{n}^{n}:=\tau_{n}$ and $M_{n}^{\prime}(b, a) \neq 0$. The function $\zeta_{k}^{n}$ is analytic and nonvanishing in $U_{k}$. Substituting equation (42) into equation (36), one gets

$$
\frac{1}{2 \pi} \int_{\Gamma_{k}} \frac{\lambda-\sigma_{k}^{n}}{\sqrt[s]{\left(\lambda_{2 k+1}-\lambda\right)\left(\lambda-\lambda_{2 k}\right)}} \zeta_{k}^{n}(\lambda) d \lambda=0 .
$$

We now drop the superscript $n$ for the remainder of this proof and write $\zeta_{k}$ as $\zeta_{k}(\lambda)=$ $\xi_{k}+\left(\zeta_{k}(\lambda)-\xi_{k}\right)$ with $\xi_{k}:=\zeta_{k}\left(\tau_{k}\right) \neq 0$. Note that

$$
\frac{1}{2 \pi} \int_{\Gamma_{k}} \frac{\lambda-\sigma_{k}}{\sqrt[s]{\left(\lambda_{2 k+1}-\lambda\right)\left(\lambda-\lambda_{2 k}\right)}} d \lambda=\tau_{k}-\sigma_{k}
$$

and hence equation (44) becomes

$$
\left(\sigma_{k}-\tau_{k}\right) \xi_{k}=\frac{1}{2 \pi} \int_{\Gamma_{k}} \frac{\left(\lambda-\sigma_{k}\right)\left(\zeta_{k}(\lambda)-\xi_{k}\right)}{\sqrt[s]{\left(\lambda_{2 k+1}-\lambda\right)\left(\lambda-\lambda_{2 k}\right)}} d \lambda
$$


To estimate the integral on the right-hand side of equation (45), note that

$$
\left|\frac{1}{2 \pi} \int_{\Gamma_{k}} \frac{f(\lambda)}{\sqrt[s]{\left(\lambda_{2 k+1}-\lambda\right)\left(\lambda-\lambda_{2 k}\right)}} d \lambda\right| \leq \max _{\lambda \in G_{k}}|f(\lambda)|
$$

for an arbitrary function $f$ analytic on $U_{k}$. We want to apply equation (46) for $f(\lambda)=$ $\left(\lambda-\sigma_{k}\right)\left(\zeta_{k}(\lambda)-\xi_{k}\right)$. Note that for $\lambda \in G_{k}$,

$$
\left|\zeta_{k}(\lambda)-\xi_{k}\right|=\left|\zeta_{k}(\lambda)-\zeta_{k}\left(\tau_{k}\right)\right| \leq M\left|\gamma_{k}\right|
$$

where $M=\sup \bigcup_{1 \leq k \leq N-1}\left\{\left|\zeta_{k}(\lambda)\right|: \lambda \in G_{k}\right\}$. Hence equation (46) leads to

$$
\left|\sigma_{k}-\tau_{k}\right|\left|\xi_{k}\right|=\sup _{\lambda \in G_{k}}\left|\lambda-\sigma_{k}\right| O\left(\gamma_{k}\right)
$$

Dividing by $\left|\xi_{k}\right| \neq 0$, we get

$$
\left|\sigma_{k}-\tau_{k}\right|=\sup _{\lambda \in G_{k}}\left|\lambda-\sigma_{k}\right| O\left(\gamma_{k}\right)
$$

and in particular, $\left|\sigma_{k}-\tau_{k}\right|=O\left(\gamma_{k}\right)$.

In the second step, we now improve estimate (47). Note that

$$
\sup _{\lambda \in G_{k}}\left|\lambda-\sigma_{k}\right| \leq\left|\sigma_{k}-\tau_{k}\right|+\sup _{\lambda \in G_{k}}\left|\lambda-\tau_{k}\right|=O\left(\gamma_{k}\right)
$$

Combining equations (47) and (48), we obtain the claimed estimate (40).

For later use, we compute the spectra of $Q(b, a)$ and $L_{2}(b, a)$ in the special case $(b, a)=\left(\beta 1_{N}, \alpha 1_{N}\right)$ with $\beta \in \mathbb{R}$ and $\alpha>0$. Here $1_{N}$ denotes the vector $(1, \ldots, 1) \in \mathbb{R}^{N}$. These points are the equilibrium points (of the restrictions) of the Toda Hamiltonian vector field (to the symplectic leaves $\mathcal{M}_{\beta, \alpha}$ ). We compute the spectrum $\left(\lambda_{j}\right)_{1 \leq j \leq 2 N}$ of the matrix $Q\left(\beta 1_{N}, \alpha 1_{N}\right)$ and the Dirichlet eigenvalues $\left(\mu_{k}\right)_{1 \leq k \leq N-1}$ of $L=L\left(\beta 1_{N}, \alpha 1_{N}\right)$ together with a normalized eigenvector $g_{l}=\left(g_{l}(j)\right)_{1 \leq j \leq N}$ of $\mu_{l}$, i.e. $L g_{l}=\mu_{l} g_{l}, g_{l}(1)=0$, and a vector $h_{l}=$ $\left(h_{l}(j)\right)_{1 \leq j \leq N}$, which is the normalized solution of $L y=\mu_{l} y$ orthogonal to $g_{l}$ satisfying $W\left(h_{l}, g_{l}\right)(N)>0$. 
Lemma 2.6. The spectrum $\left(\lambda_{j}\right)_{1 \leq j \leq 2 N}$ of $Q\left(\beta 1_{N}, \alpha 1_{N}\right)$ and the Dirichlet eigenvalues $\left(\mu_{l}\right)_{1 \leq l \leq N-1}$ of $L\left(\beta 1_{N}, \alpha 1_{N}\right)$ are given by

$$
\begin{aligned}
\lambda_{1} & =\beta-2 \alpha \\
\lambda_{2 l}=\lambda_{2 l+1}=\mu_{l} & =\beta-2 \alpha \cos \frac{l \pi}{N} \quad(1 \leq l \leq N-1), \\
\lambda_{2 N} & =\beta+2 \alpha .
\end{aligned}
$$

In particular, all spectral gaps of $Q\left(\beta 1_{N}, \alpha 1_{N}\right)$ are collapsed. For any $1 \leq l \leq N-1$, the vectors $g_{l}$ and $h_{l}$ defined by

$$
\begin{array}{ll}
g_{l}(j)=(-1)^{j+1} \sqrt{\frac{2}{N}} \sin \frac{(j-1) l \pi}{N} & (1 \leq j \leq N), \\
h_{l}(j)=(-1)^{j} \sqrt{\frac{2}{N}} \cos \frac{(j-1) l \pi}{N} & (1 \leq j \leq N)
\end{array}
$$

satisfy $L y=\mu_{l} y$ and the normalization conditions

$$
\begin{gathered}
\sum_{j=1}^{N} g_{l}(j)^{2}=\sum_{j=1}^{N} h_{l}(j)^{2}=1, \quad g_{l}(0)>0, \quad g_{l}(1)=0 \\
W\left(h_{l}, g_{l}\right)(N)>0, \quad\left\langle h_{l}, g_{l}\right\rangle_{\mathbb{R}^{N}}=0 .
\end{gathered}
$$

Remark 2.7. For $(b, a)=\left(\beta 1_{N}, \alpha 1_{N}\right)$, the fundamental solutions $y_{1}$ and $y_{2}$ are given by

$$
\begin{aligned}
& y_{1}(j, \lambda)=-\frac{\sin (\rho(j-1))}{\sin \rho} \quad(j \in \mathbb{Z}), \\
& y_{2}(j, \lambda)=\frac{\sin (\rho j)}{\sin \rho} \quad(j \in \mathbb{Z}),
\end{aligned}
$$

where $\pi<\rho<2 \pi$ is determined by $\cos \rho=\frac{\lambda-\beta}{2 \alpha}$.

Proof. For any $\lambda \in \mathbb{R}$, the difference equation (11) for $\left(\beta 1_{N}, \alpha 1_{N}\right)$ reads

$$
\left(R_{\beta, \alpha} y\right)(k):=\beta y(k)+\alpha y(k-1)+\alpha y(k+1)=\lambda y(k)
$$

and can be written as

$$
y(k-1)+y(k+1)=\frac{\lambda-\beta}{\alpha} y(k) .
$$


Since we are looking for periodic solutions of equation (54), we make the ansatz $y(k)=$ $e^{ \pm i \rho k}$. This leads to the characteristic equation

$$
2 \cos \rho \equiv e^{i \rho}+e^{-i \rho}=\frac{\lambda-\beta}{\alpha} .
$$

For the solution to be $2 N$-periodic, it is required that $\rho \in \frac{\pi}{N} \mathbb{Z}$. To put the eigenvalues in ascending order, introduce $\rho_{l}=\left(1+\frac{l}{N}\right) \pi$ with $0 \leq l \leq N$. Then for any $1 \leq j \leq 2 N$, there exists $0 \leq l \leq N$ such that

$$
\lambda_{j}=\beta+2 \alpha \cos \rho_{l}=\beta-2 \alpha \cos \frac{l \pi}{N} .
$$

Note that for $l=0, \lambda_{1}=\beta-2 \alpha$ is an eigenvalue of $Q\left(\beta 1_{N}, \alpha 1_{N}\right)$ with eigenvector $y(k)=$ $e^{i \pi k}=(-1)^{k}$. Similarly, for $l=N, \lambda_{2 N}=\beta+2 \alpha$ is an eigenvalue with eigenvector $y(k) \equiv 1$. For the eigenvalue $\lambda_{2 l}=\beta-2 \alpha \cos \frac{l \pi}{N}(1 \leq l \leq N-1)$,

$$
y_{ \pm}(k)=e^{ \pm i \rho_{l} k}
$$

are two linearly independent eigenvectors. As there are $2 N$ eigenvalues altogether, $\lambda_{2 l}$ is double for any $1 \leq l \leq N-1$, and $\lambda_{1}$ and $\lambda_{2 N}$ are both simple. It follows that all $N-1$ gaps are collapsed and hence $\mu_{l}=\lambda_{2 l}$ for all $1 \leq l \leq N-1$.

Turning to the computation of $g_{k}$ and $h_{k}$, one easily verifies that for any real number $\lambda \neq \pm 2 \alpha+\beta$, the fundamental solution $y_{1}(\cdot, \lambda)$ of equation (54) with $y_{1}(0, \lambda)=1$ and $y_{1}(1, \lambda)=0$ is given by

$$
y_{1}(j, \lambda)=-\frac{\sin (\rho(j-1))}{\sin \rho} \quad(j \in \mathbb{Z})
$$

where $\pi<\rho<2 \pi$ is determined by $\cos \rho=\frac{\lambda-\beta}{2 \alpha}$, thus proving equation (51). In the same way, one verifies equation (52). For $\lambda=\mu_{l}=\beta-2 \alpha \cos \frac{l \pi}{N}$, we then get

$$
\sin \left(\rho_{l}(j-1)\right)=\sin \left(\left(1+\frac{l}{N}\right) \pi(j-1)\right)=(-1)^{j+1} \sin \frac{(j-1) l \pi}{N} .
$$

In particular, $\sin \left(\rho_{l}(j-1)\right)=0$ for $j=1$ and $j=N+1$. As

$$
\sum_{j=1}^{N} \sin ^{2} \frac{(j-1) l \pi}{N}=\sum_{j=1}^{N} \cos ^{2} \frac{(j-1) l \pi}{N},
$$


and these two sums add up to $N$, one sees that

$$
\sum_{j=1}^{N} \sin ^{2} \frac{(j-1) l \pi}{N}=\frac{N}{2}
$$

yielding the claimed formula (49) for $g_{l}$.

By the same argument, one shows that $\tilde{h}_{l}$ given by $(-1)^{j} \sqrt{\frac{2}{N}} \cos \frac{(j-1) l \pi}{N}$ (i.e. the right side of equation (50)) satisfies $R_{\beta, \alpha} \tilde{h}_{l}=\mu_{l} \tilde{h}_{l}$ and the normalization condition $\sum_{j=1}^{N} \tilde{h}_{l}(j)^{2}=$ 1. Using standard trigonometric identities one verifies that

$$
\left\langle g_{l}, \tilde{h}_{l}\right\rangle=\sum_{j=1}^{N} g_{l}(j) \tilde{h}_{l}(j)=0
$$

and $W\left(\tilde{h}_{l}, g_{l}\right)(N)$ can be computed to be

$$
\tilde{h}_{l}(N) g_{l}(N+1)-\tilde{h}_{l}(N+1) g_{l}(N)=-\tilde{h}_{l}(N+1) g_{l}(N)=-\tilde{h}_{l}(1) g_{l}(0)>0
$$

Hence $\tilde{h}_{l}$ is indeed the eigenvector with the required normalization, i.e. $h_{l}=\tilde{h}_{l}$, thus proving equation (50).

\section{Action Variables}

In the next two sections, we define the candidates for action-angle variables on the phase space $\mathcal{M}$ and investigate some of their properties. In this section, we introduce globally defined action variables $\left(I_{n}\right)_{1 \leq n \leq N-1}$ as proposed by Flaschka-McLaughlin [5].

Definition 3.1. Let $(b, a) \in \mathcal{M}$. For $1 \leq n \leq N-1$,

$$
I_{n}:=\frac{1}{2 \pi} \int_{\Gamma_{n}} \lambda \frac{\dot{\Delta}_{\lambda}}{\sqrt[c]{\Delta_{\lambda}^{2}-4}} d \lambda
$$

where $\dot{\Delta}_{\lambda}=\frac{d}{d \lambda} \Delta_{\lambda}$ is the $\lambda$-derivative of the discriminant $\Delta_{\lambda}=\Delta(\lambda, b, a)$ and the contour $\Gamma_{n}$ and the canonical root $\sqrt[c]{\cdot}$ are given as in Section 2.

Remark 3.2. The contours $\Gamma_{n}$ can be chosen locally independently of $(b, a)$. In view of the fact that $\Delta_{\lambda}$ is a spectral invariant of $L(b, a)$, the actions $I_{n}$ are entirely determined 
by the spectrum of $L(b, a)$. In particular, $\left(I_{n}\right)_{1 \leq n \leq N-1}$ are constants of motion, since by Proposition 2.1, the Toda flow is isospectral.

Remark 3.3. The variables $\left(I_{n}\right)_{1 \leq n \leq N-1}$ can also be represented as integrals on the Riemann surface $\Sigma_{b, a}$. If $c_{n}$ denotes the lift of $\Gamma_{n}$ to the canonical sheet of $\Sigma_{b, a}$, equation (56) becomes

$$
I_{n}=\frac{1}{2 \pi} \int_{C_{n}} \lambda \frac{\dot{\Delta}_{\lambda}}{\sqrt{\Delta_{\lambda}^{2}-4}} d \lambda \quad(1 \leq n \leq N-1)
$$

From definition (56), the following result can be deduced.

Proposition 3.4. On the real space $\mathcal{M}$, each function $I_{n}$ is real, non-negative, and it vanishes if $\gamma_{n}=0$.

Proof. Since

$$
\int_{\Gamma_{n}} \frac{\dot{\Delta}_{\lambda}}{\sqrt[c]{\Delta_{\lambda}^{2}-4}} d \lambda=0
$$

it follows that

$$
I_{n}=\frac{1}{2 \pi} \int_{\Gamma_{n}}\left(\lambda-\dot{\lambda}_{n}\right) \frac{\dot{\Delta}_{\lambda}}{\sqrt[c]{\Delta_{\lambda}^{2}-4}} d \lambda
$$

By shrinking the contour of integration to the real interval, we get

$$
I_{n}=\frac{1}{\pi} \int_{\lambda_{2 n}}^{\lambda_{2 n+1}}(-1)^{N+n-1}\left(\lambda-\dot{\lambda}_{n}\right) \frac{\dot{\Delta}_{\lambda}}{\sqrt[+]{\Delta_{\lambda}^{2}-4}} d \lambda
$$

by taking into account definition (32) of the $c$-root. Since $\operatorname{sign}\left(\lambda-\dot{\lambda}_{n}\right) \dot{\Delta}_{\lambda}=(-1)^{N+n-1}$ on $\left[\lambda_{2 n}, \lambda_{2 n+1}\right] \backslash\left\{\dot{\lambda}_{n}\right\}$, the integrand is real and non-negative, hence $I_{n}$ is real and non-negative on $\mathcal{M}$, as claimed.

If $\gamma_{n}=0$, then $\lambda_{2 n}=\lambda_{2 n+1}$. Hence $\dot{\lambda}_{n}=\lambda_{2 n}=\lambda_{2 n+1}=\tau_{n}$ and

$$
\lambda-\dot{\lambda}_{n}=i \sqrt[s]{\left(\lambda_{2 n+1}-\lambda\right)\left(\lambda-\lambda_{2 n}\right)}
$$


Therefore the integrand in equation (56) is holomorphic in the interior of the contour $\Gamma_{n}$, and by Cauchy's theorem the integral in equation (56) vanishes.

The action variables $\left(I_{n}\right)_{1 \leq n \leq N-1}$ can be extended in a straightforward way to a complex neighborhood $\mathcal{W}$ of $\mathcal{M}$ in $\mathcal{M}^{\mathbb{C}}$.

Theorem 3.5. There exists a complex neighborhood $\mathcal{W}$ of $\mathcal{M}$ in $\mathcal{M}^{\mathbb{C}}$ such that for all $1 \leq$ $n \leq N-1$, the functions $I_{n}$ defined by equation (56) extend analytically to $\mathcal{W}, I_{n}: \mathcal{W} \rightarrow \mathbb{C}$.

Proof. Let $\mathcal{W}$ denote a neighborhood of $\mathcal{M}$ in $\mathcal{M}^{\mathbb{C}}$ of Lemma 2.3 and define for any $1 \leq n \leq N-1$ the functions $I_{n}$ on $\mathcal{W}$ by formula (56). Let $(b, a) \in \mathcal{W}$ be given. Then there exists a neighborhood $\mathcal{W}_{b, a}$ of $(b, a)$ in $\mathcal{W}$, so that the integration contours $\Gamma_{n}$ in formula (56) can be chosen to be the same for any element in $\mathcal{W}_{b, a}$ and $\dot{\Delta}_{\lambda} / \sqrt[c]{\Delta_{\lambda}^{2}-4}$ is analytic on $B_{\varepsilon}\left(\Gamma_{n}\right) \times \mathcal{W}_{b, a}$, where $B_{\varepsilon}\left(\Gamma_{n}\right):=\left\{\lambda \in \mathbb{C} \mid \operatorname{dist}\left(\lambda, \Gamma_{n}\right)<\varepsilon\right\}$ is the $\varepsilon$-neighborhood of $\Gamma_{n}$ with $\varepsilon$ sufficiently small. This shows that $I_{n}$ is analytic on $\mathcal{W}$.

Proposition 3.6. There exists a complex neighborhood $\mathcal{W}$ of $\mathcal{M}$ in $\mathcal{M}^{\mathbb{C}}$ such that for any $1 \leq n \leq N-1$, the quotient $I_{n} / \gamma_{n}^{2}$ extends analytically from $\mathcal{M} \backslash D_{n}$ to all of $\mathcal{W}$ and has strictly positive real part on $\mathcal{W}$. As a consequence, $\xi_{n}=\sqrt[+]{2 I_{n} / \gamma_{n}^{2}}$ is an analytic and nonvanishing function on $\mathcal{W}$, where $\sqrt[+]{ }$. is the principal branch of the square root on $\mathbb{C} \backslash(-\infty, 0]$.

Proof. Let $\mathcal{W}$ be the complex neighborhood of Theorem 3.5. Substituting equation (34) into equation (58) leads to the following identity on $\mathcal{W} \backslash D_{n}$ :

$$
I_{n}=\frac{N}{2 \pi} \int_{\Gamma_{n}} \frac{\left(\lambda-\dot{\lambda}_{n}\right)^{2}}{\sqrt[s]{\left(\lambda_{2 n+1}-\lambda\right)\left(\lambda-\lambda_{2 n}\right)}} \chi_{n}(\lambda) d \lambda,
$$

where $\chi_{n}$ is given by equation (35). Upon the substitution $\lambda(\zeta)=\tau_{n}+\frac{\gamma_{n}}{2} \zeta$, with $\tau_{n}=\frac{1}{2}\left(\lambda_{2 n}+\right.$ $\left.\lambda_{2 n+1}\right)$ and $\delta_{n}=\frac{2\left(\dot{\lambda}_{n}-\tau_{n}\right)}{\gamma_{n}}$, one then obtains

$$
\frac{2 I_{n}}{\gamma_{n}^{2}}=\frac{N}{4 \pi} \int_{\Gamma_{n}^{\prime}} \frac{\left(\zeta-\delta_{n}\right)^{2}}{\sqrt[s]{1-\zeta^{2}}} \chi_{n}\left(\tau_{n}+\frac{\gamma_{n}}{2} \zeta\right) d \zeta,
$$


where $\Gamma_{n}^{\prime}$ is the pullback of $\Gamma_{n}$ under the substitution $\lambda=\lambda(\zeta)$, i.e. a circuit in $\mathbb{C}$ around $[-1,1]$. By equation (39), $\dot{\lambda}_{n}-\tau_{n}=O\left(\gamma_{n}^{2}\right)$, and hence $\delta_{n} \rightarrow 0$ as $\gamma_{n} \rightarrow 0$. We conclude that

$$
\lim _{\gamma_{n} \rightarrow 0} \frac{2 I_{n}}{\gamma_{n}^{2}}=\frac{N}{4 \pi} \int_{\Gamma_{n}^{\prime}} \chi_{n}\left(\tau_{n}\right) \frac{\zeta^{2} d \zeta}{\sqrt[s]{1-\zeta^{2}}}=\chi_{n}\left(\tau_{n}\right) \frac{N}{2 \pi} \int_{-1}^{1} \frac{t^{2} d t}{\sqrt[+]{1-t^{2}}}=\frac{N}{4} \chi_{n}\left(\tau_{n}\right)
$$

By defining $\frac{2 I_{n}}{\gamma_{n}^{2}}$ by $\frac{N}{4} \chi_{n}\left(\tau_{n}\right)$ on $\mathcal{W} \cap D_{n}$, it follows that $\frac{2 I_{n}}{\gamma_{n}^{2}}$ is a continuous function on all of $\mathcal{W}$. This extended function is analytic on $\mathcal{W} \backslash D_{n}$ as is its restriction to $\mathcal{W} \cap D_{n}$. By Theorem A.6 in [12], it then follows that $\frac{2 I_{n}}{\gamma_{n}^{2}}$ is analytic on all of $\mathcal{W}$.

By Lemma 3.7, the quotient $I_{n} / \gamma_{n}^{2}$ can be bounded away from zero on $\mathcal{M}, \frac{I_{n}}{\gamma_{n}^{2}} \geq$ $\frac{1}{3 \pi\left(\lambda_{2 N}-\lambda_{1}\right)}$. By shrinking $\mathcal{W}$, if necessary, it then follows that for any $1 \leq n \leq N-1$, the real part of $I_{n} / \gamma_{n}^{2}$ is positive and never vanishes on $\mathcal{W}$. Hence the principal branch of the square root of $2 I_{n} / \gamma_{n}^{2}$ is well defined on $\mathcal{W}$ and $\xi_{n}$ has the claimed properties.

To show that $\sqrt[+]{\frac{2 I_{n}}{\gamma_{n}^{2}}}$ is well defined on $\mathcal{W}$, we used in the proof of Proposition 3.6 the following auxiliary result, which we prove in Appendix A.

Lemma 3.7. For any $(b, a) \in \mathcal{M}$ and any $1 \leq n \leq N-1$,

$$
\gamma_{n}^{2} \leq 3 \pi\left(\lambda_{2 N}-\lambda_{1}\right) I_{n}
$$

From definition (56), Proposition 3.4, and estimate (60), one obtains the following corollary.

Corollary 3.8. For any $(b, a) \in \mathcal{M}$ and any $1 \leq n \leq N-1$,

$$
I_{n}=0 \text { if and only if } \gamma_{n}=0
$$

Actually, Lemma 3.7 can be improved. We finish this section with an a priori estimate of the gap lengths $\gamma_{n}$ in terms of the action variables and the value of the Casimir $C_{2}$ alone, which will be shown in Appendix B.

Theorem 3.9. For any $(b, a) \in \mathcal{M}_{\beta, \alpha}$ with $\beta \in \mathbb{R}, \alpha>0$ arbitrary,

$$
\sum_{n=1}^{N-1} \gamma_{n}^{2} \leq 12 \pi^{2} \alpha\left(\sum_{n=1}^{N-1} I_{n}\right)+9 \pi^{2}(N-1)\left(\sum_{n=1}^{N-1} I_{n}\right)^{2}
$$




\section{Angle Variables}

In this section, we define and study the angle coordinates $\left(\theta_{n}\right)_{1 \leq n \leq N-1}$. Each $\theta_{n}$ is defined $\bmod 2 \pi$ on $\mathcal{W} \backslash D_{n}$, where $\mathcal{W}$ is a complex neighborhood of $\mathcal{M}$ in $\mathcal{M}^{\mathbb{C}}$ as in Lemma 2.3 and $D_{n}$ is given by equation (23).

Definition 4.1. For any $1 \leq n \leq N-1$, the function $\theta_{n}$ is defined for $(b, a) \in \mathcal{M} \backslash D_{n}$ by

$$
\theta_{n}:=\eta_{n}+\sum_{n \neq k=1}^{N-1} \beta_{k}^{n} \bmod 2 \pi
$$

where for $k \neq n$,

$$
\beta_{k}^{n}=\int_{\lambda_{2 k}}^{\mu_{k}^{*}} \frac{\psi_{n}(\lambda)}{\sqrt{\Delta_{\lambda}^{2}-4}} d \lambda, \quad \eta_{n}=\int_{\lambda_{2 n}}^{\mu_{n}^{*}} \frac{\psi_{n}(\lambda)}{\sqrt{\Delta_{\lambda}^{2}-4}} d \lambda(\bmod 2 \pi),
$$

and where for $1 \leq k \leq N-1, \mu_{k}^{*}$ is the Dirichlet divisor defined in equation (29), and $\lambda_{2 k}$ is identified with the ramification point $\left(\lambda_{2 k}, 0\right)$ on the Riemann surface $\Sigma_{b, a}$. The integration paths on $\Sigma_{b, a}$ in equation (63) are required to be admissible in the sense that their image under the projection $\pi: \Sigma_{b, a} \rightarrow \mathbb{C}$ on the first component stays inside the isolating neighborhoods $U_{k}$.

Note that, in view of the normalization conditions (36) of $\psi_{n}$, the above restriction of the paths of integration in equation (63) implies that $\eta_{n}$ and hence $\theta_{n}$ are well-defined $\bmod 2 \pi$.

Theorem 4.2. Let $\mathcal{W}$ be the complex neighborhood of $\mathcal{M}$ in $\mathcal{M}^{\mathbb{C}}$ introduced in Lemma 2.3. Then for any $1 \leq n \leq N-1$, the function $\theta_{n}: \mathcal{W} \backslash D_{n} \rightarrow \mathbb{C}(\bmod \pi)$ is analytic.

Remark 4.3. As the lexicographic ordering of the eigenvalues of $Q(b, a)$ is not continuous on $\mathcal{W}$, it follows that $\eta_{n}$ and hence $\theta_{n}$ are only continuous $\bmod \pi$ on $\mathcal{W}$.

Proof of Theorem 4.2 To see that $\theta_{n}: \mathcal{W} \backslash D_{n} \rightarrow \mathbb{C}(\bmod \pi)$ is analytic, define for any $1 \leq k \leq N-1$ the set

$$
E_{k}:=\left\{(b, a) \in \mathcal{M}^{\mathbb{C}}: \mu_{k}(b, a) \in\left\{\lambda_{2 k}(b, a), \lambda_{2 k+1}(b, a)\right\}\right\}
$$

Below, we show that for any $1 \leq k \leq N-1$ with $k \neq n, \beta_{k}^{n}$ is analytic on $\mathcal{W} \backslash\left(D_{k} \cup E_{k}\right)$, that its restrictions to $D_{k} \cap \mathcal{W}$ and $E_{k} \cap \mathcal{W}$ are weakly analytic and that it is continuous 
on $\mathcal{W}$. (Let $E$ and $F$ be complex Banach spaces, and let $U \subset E$ be open. The map $f: U \rightarrow F$ is weakly analytic on $U$, if for each $u \in U, h \in E$, and $L \in F^{*}$, the function $z \mapsto L f(u+z h)$ is analytic in some neighborhood of the origin in $\mathbb{C}$.) Together with the fact that $E_{k} \cap \mathcal{W}$ and $D_{k} \cap \mathcal{W}$ are analytic subvarieties of $\mathcal{W}$, it then follows that $\beta_{k}^{n}$ is analytic on $\mathcal{W}$-see Theorem A.6 in [12]. Similar results can be shown for $\beta_{n}^{n}=\eta_{n}(\bmod \pi)$ on $\mathcal{W} \backslash D_{n}$, and one concludes that $\theta_{n}(\bmod \pi)$ is analytic on $\mathcal{W} \backslash D_{n}$.

To prove that $\beta_{k}^{n}, k \neq n$, is analytic on $\mathcal{W} \backslash\left(D_{k} \cup E_{k}\right)$, note that since $\lambda_{2 k}$ is a simple eigenvalue on $\mathcal{W} \backslash D_{k}$, it is analytic there. Furthermore, $\mu_{k}^{*}$ is an analytic function on the (sufficiently small) neighborhood $\mathcal{W}$ of $\mathcal{M}$ in $\mathcal{M}^{\mathbb{C}}$. On $\mathcal{W} \backslash\left(D_{k} \cup E_{k}\right)$, we can use the substitution $\lambda=\lambda_{2 k}+z$ to get

$$
\beta_{k}^{n}=\int_{\lambda_{2 k}}^{\mu_{k}^{*}} \frac{\psi_{n}(\lambda)}{\sqrt{\Delta_{\lambda}^{2}-4}} d \lambda=\int_{0}^{\mu_{k}^{*}-\lambda_{2 k}} \frac{\psi_{n}\left(\lambda_{2 k}+z\right)}{\sqrt{z} \sqrt{D(z)}} d z,
$$

where $D(z)=\frac{\Delta^{2}\left(\lambda_{2 k}+z\right)-4}{z}$ is analytic near $z=0$ and $D(0) \neq 0$. Note that $D(z)$ does not vanish for $z$ on an admissible integration path not going through $\lambda_{2 k+1}$. Such a path exists, since $(b, a)$ is in the complement of $E_{k}$. Furthermore, $\psi_{n}\left(\lambda_{2 k}+z\right)$ and $D(z)$ are analytic in $z$ near such a path and depend analytically on $(b, a) \in \mathcal{W} \backslash\left(D_{k} \cup E_{k}\right)$. Combining these arguments shows that $\beta_{k}^{n}$ is analytic on $\mathcal{W} \backslash\left(D_{k} \cup E_{k}\right)$.

For $k \neq n$ with $\lambda_{2 k} \neq \lambda_{2 k+1}$, one has

$$
\int_{\lambda_{2 k}}^{\lambda_{2 k+1}} \frac{\psi_{n}(\lambda)}{\sqrt{\Delta_{\lambda}^{2}-4}} d \lambda=0
$$

As $\sigma_{k}^{n}=\lambda_{2 k}$ if $\lambda_{2 k}=\lambda_{2 k+1}$, one sees that equation (64) continues to hold for (b, $\left.a\right) \in E_{k} \cap W$ with $\lambda_{2 k}=\lambda_{2 k+1}$ and we have $\left.\beta_{k}^{n}\right|_{E_{k} \cap \mathcal{W}} \equiv 0$. To prove the analyticity of $\left.\beta_{k}^{n}\right|_{D_{k} \cap \mathcal{W}}$, consider the representation (42) of $\frac{\psi_{n}(\lambda)}{\sqrt{\Delta_{\lambda}^{2}-4}}$. For $(b, a) \in D_{k} \cap \mathcal{W}$, one has

$$
\lambda_{2 k}=\lambda_{2 k+1}=\tau_{k}=\sigma_{k}^{n},
$$

which implies that the factor $\frac{\lambda-\sigma_{k}^{n}}{\sqrt[s]{\left(\lambda_{2 k+1}-\lambda\right)\left(\lambda-\lambda_{2 k}\right)}}$ in equation (42) equals $\pm i$. Hence we can write

$$
\beta_{k}^{n}=\int_{\lambda_{2 k}}^{\mu_{k}^{*}} \frac{\psi_{n}(\lambda)}{\sqrt{\Delta_{\lambda}^{2}-4}} d \lambda= \pm i \int_{\tau_{k}}^{\mu_{k}} \zeta_{k}^{n}(\lambda) d \lambda .
$$

As $\mu_{k}$ is analytic on $\mathcal{W}$, it then follows that $\left.\beta_{k}^{n}\right|_{D_{k} \cap \mathcal{W}}$ is analytic. To see that $\beta_{k}^{n}$ is continuous on $\mathcal{W}$, one separately shows that $\beta_{k}^{n}$ is continuous at points in $\mathcal{W} \backslash\left(D_{k} \cup E_{k}\right), E_{k} \cap \mathcal{W} \backslash D_{k}$, 
$D_{k} \cap \mathcal{W} \backslash E_{k}$, and $D_{k} \cap E_{k} \cap \mathcal{W}$, where for the proof of the continuity of $\beta_{k}^{n}$ at points in $D_{k} \cap E_{k} \cap \mathcal{W}$, we use equation (42) and the estimate $\sigma_{k}^{n}-\tau_{k}=O\left(\gamma_{k}^{2}\right)$ of Lemma 2.5.

By equation (64), $\eta_{n}$ vanishes $\bmod \pi$ on $E_{n} \cap \mathcal{W} \backslash D_{n}$. Arguing in a similar way as for $\beta_{k}^{n}$, one then concludes that $\eta_{n}(\bmod \pi)$ is analytic on $\mathcal{W} \backslash D_{n}$.

\section{Gradients}

In this section, we establish formulas of the gradients of $I_{n}, \theta_{n}(1 \leq n \leq N-1)$ on $\mathcal{M}$ in terms of products of the fundamental solutions $y_{1}$ and $y_{2}$.

Consider the discriminant for a fixed value of $\lambda$ as a function on $\mathcal{M}$,

$$
\Delta_{\lambda}(b, a)=y_{1}(N)+y_{2}(N+1)
$$

Then $\Delta_{\lambda}$ is a real analytic function on $\mathcal{M}$. To obtain a formula for the gradients of $y_{1}(N)$ and $y_{2}(N+1)$ with respect to $b$, differentiate $R_{b, a} Y_{i}=\lambda y_{i}$ with respect to $b$ in the direction $v \in \mathbb{R}^{N}$ to get

$$
\left(R_{b, a}-\lambda\right)\left\langle\nabla_{b} Y_{i}, v\right\rangle(k)=-v_{k} Y_{i}(k)
$$

Differentiating $R_{b, a} Y_{i}=\lambda y_{i}$ with respect to $a$ in the direction $u \in \mathbb{R}^{N}$ leads to

$$
\left(R_{b, a}-\lambda\right)\left\langle\nabla_{a} Y_{i}, u\right\rangle(k)=-u_{k-1} Y_{i}(k-1)-u_{k} Y_{i}(k+1)
$$

Taking the sum of equations (65) and (66) yields

$$
\left(R_{b, a}-\lambda\right)\left(\left\langle\nabla_{b} Y_{i}, v\right\rangle+\left\langle\nabla_{a} Y_{i}, u\right\rangle\right)(k)=-\left(R_{v, u} Y_{i}\right)(k)
$$

which we can rewrite as

$$
\left(R_{b, a}-\lambda\right)\left\langle\nabla_{b, a} Y_{i},(v, u)\right\rangle(k)=-\left(R_{v, u} Y_{i}\right)(k)
$$

where $\langle\cdot, \cdot\rangle$ in equation (68) now denotes the standard scalar product in $\mathbb{R}^{2 N}$, whereas in equations (65), (66), and (67), it is the one in $\mathbb{R}^{N}$. The inhomogeneous Jacobi difference equation (68) for the sequence $\left\langle\nabla_{b, a} Y_{i},(v, u)\right\rangle(k)$ can be integrated using the discrete analogue of the method of the variation of constants used for inhomogeneous differential equations. As $\left\langle\nabla_{b, a} Y_{i},(v, u)\right\rangle(0)=\left\langle\nabla_{b, a} Y_{i},(v, u)\right\rangle(1)=0$, one obtains in this way for $m \geq 1$

$$
\left\langle\nabla_{b, a} Y_{i},(v, u)\right\rangle(m)=-\left(\frac{y_{2}(m)}{a_{N}} \sum_{k=1}^{m} Y_{1}(k)\left(R_{v, u} Y_{i}\right)(k)-\frac{Y_{1}(m)}{a_{N}} \sum_{k=1}^{m} Y_{2}(k)\left(R_{v, u} Y_{i}\right)(k)\right) .
$$


In the sequel, we will use equation (69) to derive various formulas for the gradients. The common feature among these formulas is that they involve products between the fundamental solutions $y_{1}$ and $y_{2}$ of equation (11). Whereas the gradients with respect to $b=\left(b_{1}, \ldots, b_{N}\right)$ involve products computed by componentwise multiplication, the gradients with respect to $a=\left(a_{1}, \ldots, a_{N}\right)$ involve products obtained by multiplying shifted components, reflecting the fact that the $b_{j}$ are the diagonal elements of the symmetric matrix $L(b, a)$, whereas the $a_{j}$ are the off-diagonal elements of $L(b, a)$.

To simplify notation for the formulas in this section, we define for sequences $\left(v(j)_{j \in \mathbb{Z}}\right),\left(w(j)_{j \in \mathbb{Z}}\right) \subseteq \mathbb{C}$ the $N$-vectors

$$
\begin{aligned}
v \cdot w & :=(v(j) w(j))_{1 \leq j \leq N}, \\
v \cdot S w & :=(v(j) w(j+1))_{1 \leq j \leq N},
\end{aligned}
$$

where $S$ denotes the shift operator of order 1. Combining equations (70) and (71), we define the $2 N$-vector

$$
v \cdot \mathbf{s} w:=(v \cdot w, v \cdot S w+w \cdot S v)
$$

In case $v=w$, we also use the shorter notation

$$
v^{2}:=v \cdot \mathbf{s} v
$$

Written componentwise, $v \cdot \mathbf{s} w$ is the $2 N$-vector

$$
(v \cdot \mathbf{s} w)(j)=\left\{\begin{array}{cc}
v(j) w(j) & (1 \leq j \leq N) \\
v(j-N) w(j-N+1)+v(j-N+1) w(j-N) & (N<j \leq 2 N) .
\end{array}\right.
$$

Proposition 5.1. For any $(b, a) \in \mathcal{M}$, the gradient $\nabla_{b, a} \Delta_{\lambda}=\left(\nabla_{b} \Delta_{\lambda}, \nabla_{a} \Delta_{\lambda}\right)$ is given by

$$
\begin{aligned}
& -a_{N} \nabla_{b} \Delta_{\lambda}=y_{2}(N) y_{1} \cdot y_{1}-y_{1}(N+1) y_{2} \cdot y_{2}+\left(y_{2}(N+1)-y_{1}(N)\right) y_{1} \cdot y_{2} \\
& -a_{N} \nabla_{a} \Delta_{\lambda}=2 y_{2}(N) y_{1} \cdot S y_{1}-2 y_{1}(N+1) y_{2} \cdot S y_{2}+\left(y_{2}(N+1)-y_{1}(N)\right)\left(y_{1} \cdot S y_{2}+y_{2} \cdot S y_{1}\right)
\end{aligned}
$$


or in the notation introduced above

$$
\nabla_{b, a} \Delta_{\lambda}=-\frac{1}{a_{N}}\left(y_{2}(N) y_{1}^{2}-y_{1}(N+1) y_{2}^{2}+\left(y_{2}(N+1)-y_{1}(N)\right) y_{1} \cdot \mathbf{s} y_{2}\right)
$$

The gradients $\nabla_{b} \Delta_{\lambda}$ and $\nabla_{a} \Delta_{\lambda}$ admit the representations $(1 \leq m \leq N)$,

$$
\begin{aligned}
& \frac{\partial \Delta_{\lambda}}{\partial b_{m}}=-\frac{1}{a_{m}} y_{2}\left(N, \lambda, S^{m} b, S^{m} a\right), \\
& \frac{\partial \Delta_{\lambda}}{\partial a_{m}}=-\left(\frac{1}{a_{m}} y_{2}\left(N+1, \lambda, S^{m} b, S^{m} a\right)+\frac{1}{a_{m+1}} y_{2}\left(N-1, \lambda, S^{m+1} b, S^{m+1} a\right)\right) .
\end{aligned}
$$

Proof. The claimed formula (76) follows from the definition of $\Delta_{\lambda}$ and formula (69). Indeed, evaluate equation (69) for $i=1$ and $m=N$ to get

$$
\begin{aligned}
\left\langle\nabla_{b, a} Y_{1},(v, u)\right\rangle(N)= & -\frac{y_{2}(N)}{a_{N}} \sum_{k=1}^{N} y_{1}(k)\left(u_{k-1} Y_{1}(k-1)+v_{k} Y_{1}(k)+u_{k Y_{1}}(k+1)\right) \\
& +\frac{y_{1}(N)}{a_{N}} \sum_{k=1}^{N} y_{2}(k)\left(u_{k-1} Y_{1}(k-1)+v_{k} Y_{1}(k)+u_{k} Y_{1}(k+1)\right) .
\end{aligned}
$$

In order to identify these two sums with $\left\langle y_{1}^{2},(v, u)\right\rangle$ and $\left\langle y_{1} \cdot \mathbf{s} y_{2},(v, u)\right\rangle$, respectively, note that

$$
\sum_{k=1}^{N} u_{k-1} y_{1}(k) y_{1}(k-1)=\sum_{k=1}^{N} u_{k} y_{1}(k) y_{1}(k+1)+u_{N} T_{1}
$$

where

$$
T_{1}:=y_{1}(0) y_{1}(1)-y_{1}(N) y_{1}(N+1)
$$

For the second sum in equation (79), we get an expression of the same type with a similar correction term

$$
T_{2}:=y_{1}(0) y_{2}(1)-y_{1}(N) y_{2}(N+1)
$$

Taking into account the initial conditions of the fundamental solutions and the Wronskian identity (13), one sees that $y_{2}(N) T_{1}-y_{1}(N) T_{2}$ vanishes. Hence we have the formula

$$
\left\langle\nabla_{b, a Y_{1}},(v, u)\right\rangle(N)=-\frac{1}{a_{N}}\left(y_{2}(N)\left\langle y_{1}^{2},(v, u)\right\rangle-y_{1}(N)\left\langle y_{1} \cdot \mathbf{s} y_{2},(v, u)\right\rangle\right)
$$


Similarly, evaluating formula (69) for $i=2$ and $m=N+1$ leads to

$$
\left\langle\nabla_{b, a} Y_{2},(v, u)\right\rangle(N+1)=-\frac{1}{a_{N}}\left(y_{2}(N+1)\left\langle y_{1} \cdot \mathbf{s} y_{2},(v, u)\right\rangle-y_{1}(N+1)\left\langle y_{2}^{2},(v, u)\right\rangle\right) .
$$

Here we used that the value of the right side of equation (69) does not change when we omit the term for $k=m=N+1$ in both sums.

It remains to prove the two formulas (77) and (78). We first note that

$y_{2}\left(n, \lambda, S^{m} b, S^{m} a\right)=\frac{a_{m}}{a_{N}}\left(y_{2}(n+m, \lambda, b, a)_{y_{1}}(m, \lambda, b, a)-y_{1}(n+m, \lambda, b, a)_{y_{2}}(m, \lambda, b, a)\right)$,

since both sides of equation (82) are solutions of $R_{S^{m} b, S^{m} a} y=\lambda y$ (for fixed $m \in \mathbb{Z}$ ) with the same initial conditions at $n=0,1$. For $n=1$, this follows from the Wronskian identity (13). Similarly, one shows that

$$
\begin{aligned}
& y_{1}(N+m, \lambda)=y_{1}(N, \lambda) y_{1}(m, \lambda)+y_{1}(N+1, \lambda) y_{2}(m, \lambda), \\
& y_{2}(N+m, \lambda)=y_{2}(N, \lambda) y_{1}(m, \lambda)+y_{2}(N+1, \lambda) y_{2}(m, \lambda)
\end{aligned}
$$

for any $(b, a) \in \mathcal{M}$. Hence, suppressing the variable $\lambda$, we get

$$
\begin{aligned}
y_{2}\left(N, S^{m} b, S^{m} a\right)= & \frac{a_{m}}{a_{N}}\left(\left(y_{2}(N) y_{1}(m)+y_{2}(N+1) y_{2}(m)\right) y_{1}(m)\right. \\
& \left.-\left(y_{1}(N) y_{1}(m)+y_{1}(N+1) y_{2}(m)\right) y_{2}(m)\right) \\
= & \frac{a_{m}}{a_{N}}\left(y_{2}(N) y_{1}(m)^{2}+\left(y_{2}(N+1)-y_{1}(N)\right) y_{1}(m) y_{2}(m)-y_{1}(N+1)_{y_{2}}(m)^{2}\right) .
\end{aligned}
$$

By equation (74), this leads to

$$
y_{2}\left(N, S^{m} b, S^{m} a\right)=-a_{m} \frac{\partial \Delta_{\lambda}}{\partial b_{m}}
$$

and formula (77) is established. To prove equation (78), we first conclude from equation (82) that

$$
\begin{aligned}
\frac{a_{N}}{a_{m+1}} y_{2}\left(N-1, S^{m+1} b, S^{m+1} a\right)= & y_{2}(N+m, b, a) y_{1}(m+1, b, a) \\
& -y_{1}(N+m, b, a) y_{2}(m+1, b, a)
\end{aligned}
$$

and

$$
\begin{aligned}
\frac{a_{N}}{a_{m}} y_{2}\left(N+1, S^{m} b, S^{m} a\right)= & y_{2}(N+m+1, b, a) y_{1}(m, b, a) \\
& -y_{1}(N+m+1, b, a) y_{2}(m, b, a) .
\end{aligned}
$$


Now expand the right-hand sides of equations (85) and (86) according to equations (83) and (84). By equation (75), the sum of equations (85) and (86) is $-a_{N} \frac{\partial \Delta_{\lambda}}{\partial a_{m}}$, thus proving equation (78).

As a next step, we compute the gradients of the Dirichlet and periodic eigenvalues. In the following lemma, we consider the fundamental solution $y_{1}(\cdot, \mu)$ as an $N$-vector $y_{1}(j, \mu)_{1 \leq j \leq N}$. Let $\left\|y_{1}(\mu)\right\|^{2}=\sum_{j=1}^{N} y_{1}(j, \mu)^{2}$, and denote by $\cdot$ the derivative with respect to $\lambda$.

Lemma 5.2. If $\mu$ is a Dirichlet eigenvalue of $L(b, a)$, then

$$
a_{N} Y_{1}(N, \mu) \dot{y}_{1}(N+1, \mu)=\left\|y_{1}(\mu)\right\|^{2}>0
$$

In particular, $\dot{Y}_{1}(N+1, \mu) \neq 0$, which implies that all Dirichlet eigenvalues are simple.

Proof. This follows from adding up the relations (16).

As the Dirichlet eigenvalues $\left(\mu_{n}\right)_{1 \leq n \leq N-1}$ of $L(b, a)$ coincide with the roots of $y_{1}(N+$ $1, \mu)$ and these roots are simple, they are real analytic on $\mathcal{M}$. Similarly, the eigenvalues $\lambda_{1}$ and $\lambda_{2 N}$ are real analytic on $\mathcal{M}$, whereas for any $1 \leq n \leq N-1, \lambda_{2 n}$ and $\lambda_{2 n+1}$ are real analytic on $\mathcal{M} \backslash D_{n}$. Note that for $(b, a) \in \mathcal{M} \backslash D_{n}$ and $i \in\{2 n, 2 n+1\}$, we have $\dot{\Delta}_{\lambda_{i}} \neq 0$ as $\lambda_{i}$ is a simple eigenvalue.

Proposition 5.3. For any $1 \leq n \leq N-1$, the gradients of the periodic eigenvalues $\lambda_{i}$ $(i=2 n, 2 n+1)$ on $\mathcal{M} \backslash D_{n}$ and of the Dirichlet eigenvalues $\mu_{n}$ on $\mathcal{M}$ are given by

$$
\nabla_{b, a} \lambda_{i}=-\frac{\left.\nabla_{b, a} \Delta_{\lambda}\right|_{\lambda=\lambda_{i}}}{\dot{\Delta}_{\lambda_{i}}}=f_{i}^{2} \quad \text { and } \quad \nabla_{b, a} \mu_{n}=g_{n}^{2}
$$

where we denote by $f_{i}$ the eigenvector of $L(b, a)$ associated to $\lambda_{i}$, normalized by

$$
\sum_{j=1}^{N} f_{i}(j)^{2}=1 \quad \text { and } \quad\left(f_{i}(1), f_{i}(2)\right) \in\left(\mathbb{R}_{>0} \times \mathbb{R}\right) \cup\left(\{0\} \times \mathbb{R}_{>0}\right)
$$

and where $g_{n}=\left(g_{n}(j)\right)_{1 \leq j \leq N}$ is the fundamental solution $y_{1}\left(\cdot, \mu_{n}\right)$ normalized, so that $\sum_{j=1}^{N} g_{n}(j)^{2}=1$. 
Proof. We first show the second formula in equation (88). Differentiating $y_{1}\left(N+1, \mu_{n}\right)=$ 0 with respect to $(b, a)$, one obtains

$$
\nabla_{b, a} \mu_{n}=-\frac{\left.\nabla_{b, a} Y_{1}(N+1, \lambda)\right|_{\lambda=\mu_{n}}}{\dot{Y}_{1}\left(N+1, \mu_{n}\right)} .
$$

Here we used that $\dot{y}_{1}\left(N+1, \mu_{n}\right) \neq 0$ by Lemma 5.2. To compute the gradient $\nabla_{b, a} Y_{1}(N+$ $1, \lambda)\left.\right|_{\lambda=\mu_{n}}$, we evaluate equation (69) for $i=1$ and $m=N+1$. In view of $y_{1}\left(N+1, \mu_{n}\right)=0$ and taking into account equation (26), one then gets

$$
\nabla_{b, a} \mu_{n}=\frac{y_{1}^{2}\left(\mu_{n}\right)}{a_{N} Y_{1}\left(N, \mu_{n}\right) \dot{y}_{1}\left(N+1, \mu_{n}\right)} .
$$

The claimed formula $\nabla_{b, a} \mu_{n}=g_{n}^{2}$ now follows from Lemma 5.2. By differentiating $\Delta_{\lambda_{i}}=$ \pm 2 with respect to $(b, a)$, one obtains $\nabla_{b, a} \lambda_{i}=-\left.\nabla_{b, a} \Delta_{\lambda}\right|_{\lambda=\lambda_{i}} / \dot{\Delta}_{\lambda_{i}}$ in a similar fashion. To see that $\nabla_{b, a} \lambda_{i}=f_{i}^{2}$, differentiate $R_{b, a} f_{i}=\lambda_{i} f_{i}$ with respect to $(b, a)$ in the direction $(v, u) \in$ $\mathbb{R}^{2 N}$,

$$
R_{b, a}\left\langle\nabla_{b, a} f_{i},(v, u)\right\rangle(k)+\left(R_{v, u} f_{i}\right)(k)=\left\langle\nabla_{b, a} \lambda_{i},(v, u)\right\rangle f_{i}(k)+\lambda_{i}\left\langle\nabla_{b, a} f_{i},(v, u)\right\rangle(k),
$$

where $\langle\cdot, \cdot\rangle$ denotes the standard scalar product in $\mathbb{R}^{2 N}$. Take the scalar product (in $\mathbb{R}^{N}$ ) of the above equation with $f_{i}$. Now use that

$$
\left\langle\nabla_{b, a} f_{i}(v, u), R_{b, a} f_{i}\right\rangle_{\mathbb{R}^{N}}=\lambda_{i}\left\langle\nabla_{b, a} f_{i}(v, u), f_{i}\right\rangle_{\mathbb{R}^{N}}
$$

$\left\langle f_{i}, f_{i}\right\rangle_{\mathbb{R}^{N}}=1$, and

$$
\left\langle R_{v, u} f_{i}, f_{i}\right\rangle_{\mathbb{R}^{N}}=\left\langle f_{i}^{2},(v, u)\right\rangle_{\mathbb{R}^{2 N}}
$$

to conclude that $\nabla_{b, a} \lambda_{i}=f_{i}^{2}$ holds.

To compute the Poisson brackets involving angle variables, we need to establish some additional auxiliary results. Recall from Section 3 that for $1 \leq k, n \leq N-1$ with $k \neq n$ and $(b, a) \in \mathcal{M}, \beta_{k}^{n}$ is given by

$$
\beta_{k}^{n}=\int_{\lambda_{2 k}}^{\mu_{k}^{*}} \frac{\psi_{n}(\lambda)}{\sqrt{\Delta_{\lambda}^{2}-4}} d \lambda
$$


whereas

$$
\beta_{n}^{n}:=\eta_{n}=\int_{\lambda_{2 n}}^{\mu_{n}^{*}} \frac{\psi_{n}(\lambda)}{\sqrt{\Delta_{\lambda}^{2}-4}} d \lambda(\bmod 2 \pi)
$$

By Theorem 4.2, the functions $\beta_{k}^{n}$ with $k \neq n$ are real analytic on $\mathcal{M}$, whereas $\beta_{n}^{n}$, when considered $\bmod \pi$, is real analytic on $\mathcal{M} \backslash D_{n}$.

Proposition 5.4. Let $1 \leq k \leq N-1$ and $(b, a) \in \mathcal{M}$. If $\gamma_{k}>0$ and $\lambda_{2 k}=\mu_{k}$, then for any $1 \leq n \leq N-1$,

$$
\nabla_{b, a} \beta_{k}^{n}=-\frac{\psi_{n}\left(\mu_{k}\right)}{a_{N} \dot{\Delta}_{\mu_{k}}} g_{k} \cdot \mathbf{s} h_{k}
$$

where $h_{k}$ denotes the solution of $R_{b, a} Y=\mu_{k} y$ orthogonal to $g_{k}$, that is,

$$
\sum_{j=1}^{N} g_{k}(j) h_{k}(j)=0
$$

satisfying the normalization condition $W\left(h_{k}, g_{k}\right)(N)=1$.

Proof. We use a limiting procedure first introduced in [15] for the nonlinear Schrödinger equation and subsequently used for the $\mathrm{KdV}$ equation in [11], [12]. We approximate $(b, a) \in \mathcal{M}$ with $\lambda_{2 k}(b, a)=\mu_{k}(b, a)<\lambda_{2 k+1}(b, a)$ by $\left(b^{\prime}, a^{\prime}\right) \in \operatorname{Iso}(b, a)$, satisfying $\lambda_{2 k}(b, a)<$ $\mu_{k}\left(b^{\prime}, a^{\prime}\right)<\lambda_{2 k+1}(b, a)$. For such $\left(b^{\prime}, a^{\prime}\right)$, using the substitution $\lambda=\lambda_{2 k}+z$ in the integral of equation (91), we obtain

$$
\beta_{k}^{n}\left(b^{\prime}, a^{\prime}\right)=\int_{\lambda_{2 k}}^{\mu_{k}^{*}} \frac{\psi_{n}(\lambda)}{\sqrt{\Delta_{\lambda}^{2}-4}} d \lambda=\int_{0}^{\mu_{k}-\lambda_{2 k}} \frac{\psi_{n}\left(\lambda_{2 k}+z\right)}{\sqrt{z} \sqrt{D(z)}} d z
$$

where $D(z) \equiv D\left(\lambda_{2 k}, z\right):=\left(\Delta^{2}\left(\lambda_{2 k}+z\right)-4\right) / z$. Taking the gradient, undoing the substitution, and recalling definition (29) of the starred square root then leads to

$$
\nabla_{b, a} \beta_{k}^{n}=\frac{\psi_{n}\left(\mu_{k}\right)}{\sqrt[*]{\Delta_{\mu_{k}}^{2}-4}}\left(\nabla_{b, a} \mu_{k}-\nabla_{b, a} \lambda_{2 k}\right)+E\left(b^{\prime}, a^{\prime}\right),
$$


with the remainder term $E\left(b^{\prime}, a^{\prime}\right)$ given by

$$
E\left(b^{\prime}, a^{\prime}\right)=\int_{0}^{\mu_{k}-\lambda_{2 k}} \nabla_{b^{\prime}, a^{\prime}}\left(\frac{\psi_{n}\left(\lambda_{2 k}+z\right)}{\sqrt{D\left(\lambda_{2 k}, z\right)}}\right) \frac{d z}{\sqrt{z}} .
$$

As the gradient in the latter integral is a bounded function in $z$ near $z=0$, locally uniformly in $\left(b^{\prime}, a^{\prime}\right)$, it follows from the dominated convergence theorem that $\lim _{\left(b^{\prime}, a^{\prime}\right) \rightarrow(b, a)} E\left(b^{\prime}, a^{\prime}\right)=0$.

The gradient $\nabla_{b, a} \beta_{k}^{n}$ depends continuously on $(b, a) \in \mathcal{M}$, hence we can conclude by equation (94) that it can be written as

$$
\nabla_{b, a} \beta_{k}^{n}=\lim _{\left(b^{\prime}, a^{\prime}\right) \rightarrow(b, a)} \frac{\psi_{n}\left(\mu_{k}\right)}{\sqrt[*]{\Delta_{\mu_{k}}^{2}-4}}\left(\nabla_{b^{\prime}, a^{\prime}} \mu_{k}-\nabla_{b^{\prime}, a^{\prime}} \lambda_{2 k}\right)
$$

The gradient of both sides of the Wronskian identity (13)

$$
y_{1}(N, \lambda) y_{2}(N+1, \lambda)-y_{1}(N+1, \lambda) y_{2}(N, \lambda)=1
$$

leads to

$$
y_{1}(N+1) \nabla_{b, a} Y_{2}(N)+y_{2}(N) \nabla_{b, a} Y_{1}(N+1)=y_{2}(N+1) \nabla_{b, a} \Delta+\left(y_{1}(N)-y_{2}(N+1)\right) \nabla_{b, a} Y_{2}(N+1) .
$$

The $\lambda$-derivative can then be computed to be

$$
y_{1}(N+1) \dot{y}_{2}(N)+\dot{y}_{1}(N+1) y_{2}(N)=y_{2}(N+1) \dot{\Delta}+\left(y_{1}(N)-y_{2}(N+1)\right) \dot{y}_{2}(N+1) .
$$

Using equations (96), (97), and $y_{1}\left(N+1, \mu_{k}\right)=0$, formula (89) for $\nabla_{b, a} \mu_{k}$ leads to

$$
\nabla_{b, a} \mu_{k}=-\left.\frac{y_{2}(N+1) \nabla_{b, a} \Delta+\left(y_{1}(N)-y_{2}(N+1)\right) \nabla_{b, a} y_{2}(N+1)}{y_{2}(N+1) \dot{\Delta}+\left(y_{1}(N)-y_{2}(N+1)\right) \dot{y}_{2}(N+1)}\right|_{\mu_{k}} .
$$

Further by equation (88),

$$
\nabla_{b, a} \lambda_{2 k}=-\left.\frac{\nabla_{b, a} \Delta}{\dot{\Delta}}\right|_{\lambda=\lambda_{2 k}}
$$


Now substitute equations (98) and (99) into $\left(\nabla_{b^{\prime}, a^{\prime}} \mu_{k}-\nabla_{b^{\prime}, a^{\prime}} \lambda_{2 k}\right)$ and use that by equation (29),

$$
\sqrt[*]{\Delta_{\mu_{k}}^{2}-4}=\left.\left(y_{1}(N)-y_{2}(N+1)\right)\right|_{\mu_{k}} .
$$

We claim that

$$
\lim _{\substack{\left(b^{\prime}, a^{\prime}\right) \\ \rightarrow(b, a)}} \frac{\nabla_{b, a} \mu_{k}-\nabla_{b, a} \lambda_{2 k}}{\sqrt[*]{\Delta_{\mu_{k}}^{2}-4}}=\left.\frac{\dot{Y}_{2}(N+1) \nabla_{b, a} Y_{1}(N)-\dot{Y}_{1}(N) \nabla_{b, a} Y_{2}(N+1)}{\dot{\Delta} \dot{Y}_{1}(N+1) Y_{2}(N)}\right|_{\lambda_{2 k}} .
$$

Indeed, to obtain equation (100) after the above-mentioned substitutions, we split the fraction $\frac{1}{\sqrt[*]{\Delta_{\mu_{k}}^{2}-4}}\left(\nabla_{b^{\prime}, a^{\prime}} \mu_{k}-\nabla_{b^{\prime}, a^{\prime}} \lambda_{2 k}\right)$ into two parts which are treated separately. In the first part, we collect all terms in $\frac{1}{\sqrt[*]{\Delta_{\mu_{k}}^{2}-4}}\left(\nabla_{b^{\prime}, a^{\prime}} \mu_{k}-\nabla_{b^{\prime}, a^{\prime}} \lambda_{2 k}\right)$ which contain $\left.\left(y_{1}(N)-y_{2}(N+1)\right)\right|_{\mu_{k}}$ in the nominator, and get after cancellation,

$$
I\left(a^{\prime}, b^{\prime}\right):=\frac{-\left.\left.\dot{\Delta}\right|_{\lambda_{2 k}} \cdot \nabla_{b^{\prime}, a^{\prime} Y_{2}}(N+1)\right|_{\mu_{k}}+\left.\left.\nabla_{b^{\prime}, a^{\prime}} \Delta\right|_{\lambda_{2 k}} \cdot \dot{y}_{2}(N+1)\right|_{\mu_{k}}}{\left.\left.\dot{\Delta}\right|_{\lambda_{2 k}} \cdot\left(y_{2}(N+1) \dot{\Delta}+\left(y_{1}(N)-y_{2}(N+1)\right) \dot{y}_{2}(N+1)\right)\right|_{\mu_{k}}} .
$$

Using again equation (97), we then get

$$
\lim _{\left(b^{\prime}, a^{\prime}\right) \rightarrow(b, a)} I\left(b^{\prime}, a^{\prime}\right)=\left.\frac{\dot{y}_{2}(N+1) \nabla_{b, a} Y_{1}(N)-\dot{Y}_{1}(N) \nabla_{b, a} Y_{2}(N+1)}{\dot{\Delta} \dot{Y}_{1}(N+1)_{2}(N)}\right|_{\lambda_{2 k}} .
$$

The second term is then given by

$$
I I\left(b^{\prime}, a^{\prime}\right)=\frac{\left.y_{2}(N+1)\right|_{\mu_{k}} \cdot\left(\left.\left.\nabla_{b^{\prime}, a^{\prime}} \Delta\right|_{\lambda_{2 k}} \cdot \dot{\Delta}\right|_{\mu_{k}}-\left.\left.\dot{\Delta}\right|_{\lambda_{2 k}} \cdot \nabla_{b^{\prime}, a^{\prime}} \Delta\right|_{\mu_{k}}\right)}{\left.\left.\left.\dot{\Delta}\right|_{\lambda_{2 k}} \cdot\left(y_{1}(N)-y_{2}(N+1)\right)\right|_{\mu_{k}} \cdot\left(\dot{y}_{1}(N+1) Y_{2}(N)\right)\right|_{\mu_{k}}} .
$$

Note that the nominator of $I I\left(b^{\prime}, a^{\prime}\right)$ is of the order $O\left(\mu_{k}-\lambda_{2 k}\right)$. In view of equation (29), we have

$$
\left.\left(y_{1}(N)-y_{2}(N+1)\right)\right|_{\mu_{k}}=O\left(\sqrt{\mu_{k}-\lambda_{2 k}}\right),
$$

whereas the other terms in the denominator of $I I\left(b^{\prime}, a^{\prime}\right)$ are bounded away from zero. Indeed, $\lambda_{2 k}$ being a simple eigenvalue for $(b, a)$ means $\left.\dot{\Delta}\right|_{\lambda_{2 k}} \neq 0$ for $\left(b^{\prime}, a^{\prime}\right)$ near $(b, a)$. Further, use a version of equation (97) in the case $\lambda_{2 k}=\mu_{k}$ to conclude that

$$
\dot{Y}_{1}(N+1)_{y_{2}}(N)=y_{2}(N+1) \dot{\Delta}_{\lambda_{2 k}}
$$


Hence $\left.\dot{y}_{1}(N+1) y_{2}(N)\right|_{\mu_{k}} \neq 0$ for $\left(b^{\prime}, a^{\prime}\right)$ near $(b, a)$ and $I I\left(b^{\prime}, a^{\prime}\right)$ vanishes in the limit of $\mu_{k} \rightarrow \lambda_{2 k}$.

Substituting equations (80) and (81) into equation (100), we obtain

$$
\left.\frac{\dot{Y}_{2}(N+1) \nabla_{b, a} Y_{1}(N)-\dot{Y}_{1}(N) \nabla_{b, a} Y_{2}(N+1)}{\dot{\Delta} \dot{Y}_{1}(N+1) y_{2}(N)}\right|_{\mu_{k}}=-\frac{1}{a_{N} \dot{\Delta}} Y_{1} \cdot \mathbf{s} Y_{0}
$$

with $y_{0}=\frac{\dot{y}_{2}(N+1)}{\dot{y}_{1}(N+1)} Y_{1}-Y_{2}$. Hence

$$
\nabla_{b, a} \beta_{k}^{n}=-\frac{\psi_{n}\left(\mu_{k}\right)}{a_{N} \dot{\Delta}\left(\mu_{k}\right)} y_{1} \cdot \mathbf{s} y_{0}
$$

Since $\beta_{k}^{n}$ is invariant under the translation $b \mapsto b+t(1, \ldots, 1)$, the scalar product $\left\langle\nabla_{b, a} \beta_{k}^{n},(\mathbf{1}, \mathbf{0})\right\rangle_{\mathbb{R}^{2 N}}$ vanishes. Hence

$$
0=\sum_{j=1}^{N} \frac{\partial \beta_{k}^{n}}{\partial b_{j}}=-\frac{\psi_{n}\left(\mu_{k}\right)}{a_{N} \dot{\Delta}\left(\mu_{k}\right)} \sum_{j=1}^{N} y_{1}(j) y_{0}(j)
$$

It means that $y_{1}$ and $y_{0}$ are orthogonal to each other. Finally, we introduce $h_{k}:=\left\|y_{1}\right\| y_{0}$ and verify that

$$
W\left(h_{k}, g_{k}\right)=W\left(\left\|y_{1}\right\| y_{0}, \frac{y_{1}}{\left\|y_{1}\right\|}\right)=W\left(y_{0}, y_{1}\right)=W\left(\frac{\dot{y}_{2}(N+1)}{\dot{y}_{1}(N+1)} y_{1}-y_{2}, y_{1}\right)
$$

By equation (13), it then follows that

$$
W\left(h_{k}, g_{k}\right)=-W\left(y_{2}, y_{1}\right)=W\left(y_{1}, y_{2}\right)
$$

Hence by equation (14),

$$
W\left(h_{k}, g_{k}\right)(N)=W\left(y_{1}, y_{2}\right)(N)=1
$$

This completes the proof of Proposition 5.4.

\section{Orthogonality Relations}

In Propositions 5.1, 5.3, and 5.4, we have expressed the gradients of $\Delta_{\lambda}, \mu_{n}$, and, on a subset of $\mathcal{M}$, of $\beta_{k}^{n}$ in terms of products of fundamental solutions of the difference equation 
(11). In this section, we establish orthogonality relations between such products — see [2] for similar computations. Recall that in equation (72), we have introduced for arbitrary sequences $\left(v_{j}\right)_{j \in \mathbb{Z}},\left(w_{j}\right)_{j \in \mathbb{Z}}$ the $2 N$-vector $v \cdot \mathbf{s} w$.

Lemma 6.1. For any $(b, a) \in \mathcal{M}$, let $v_{1}, w_{1}$ and $v_{2}, w_{2}$ be pairs of solutions of equation (11) for arbitrarily given real numbers $\mu$ and $\lambda$, respectively. Then

$$
\frac{2(\lambda-\mu)}{a_{1} a_{N}}\left\langle v_{1} \cdot \mathbf{s} w_{1}, J\left(v_{2} \cdot \mathbf{s} w_{2}\right)\right\rangle=V+B
$$

where

$$
V:=\left.\left(W_{1} \cdot S W_{2}\right)\right|_{0} ^{N}+\left.\left(S W_{1} \cdot W_{2}\right)\right|_{0} ^{N}
$$

with $W_{1}$ and $W_{2}$ denoting the Wronskians $W_{1}:=W\left(v_{1}, w_{2}\right), W_{2}:=W\left(w_{1}, v_{2}\right)$, and where $B$ is given by

$$
B:=\frac{(\lambda-\mu)}{a_{1}}\left(\left.\left(v_{1} \cdot w_{1}\right)\right|_{1} ^{N+1}\left(v_{2} \cdot \mathbf{s} w_{2}\right)(2 N)-\left.\left(v_{2} \cdot w_{2}\right)\right|_{1} ^{N+1}\left(v_{1} \cdot \mathbf{s} w_{1}\right)(2 N)\right)
$$

Proof. We prove equation (101) by a straightforward calculation, using the recurrence property (15) of the Wronskian sequences $W_{1}$ and $W_{2}$. By definition (3) of $J$, we can write

$$
2\left\langle v_{1} \cdot \mathbf{s} w_{1}, J\left(v_{2} \cdot \mathbf{s} w_{2}\right)\right\rangle=E_{1}+B_{1},
$$

where

$$
\begin{aligned}
E_{1}:= & \sum_{k=1}^{N} a_{k}\left[\left(v_{1} \cdot \mathbf{s} w_{1}\right)(k)\left(v_{2} \cdot \mathbf{s} w_{2}\right)(N+k)-\left(v_{1} \cdot \mathbf{s} w_{1}\right)(k+1)\left(v_{2} \cdot \mathbf{s} w_{2}\right)(N+k)\right. \\
& \left.-\left(v_{1} \cdot \mathbf{s} w_{1}\right)(N+k)\left(v_{2} \cdot \mathbf{s} w_{2}\right)(k)+\left(v_{1} \cdot \mathbf{s} w_{1}\right)(N+k)\left(v_{2} \cdot \mathbf{s} w_{2}\right)(k+1)\right]
\end{aligned}
$$

and

$$
\begin{aligned}
B_{1}:= & a_{N}\left(\left(v_{1} \cdot \mathbf{s} w_{1}\right)(N+1)-\left(v_{1} \cdot \mathbf{s} w_{1}\right)(1)\right)\left(v_{2} \cdot \mathbf{s} w_{2}\right)(2 N) \\
& +a_{N}\left(\left(v_{2} \cdot \mathbf{s} w_{2}\right)(1)-\left(v_{2} \cdot \mathbf{s} w_{2}\right)(N+1)\right)\left(v_{1} \cdot \mathbf{s} w_{1}\right)(2 N)
\end{aligned}
$$


Let us first consider $E_{1}$. Calculating the products $v_{j} \cdot \mathbf{s} w_{j}$ according to equation (72), we obtain, after regrouping,

$$
\begin{aligned}
E_{1}= & \sum_{k=1}^{N} a_{k}\left[\left(v_{2}(k) w_{1}(k)+v_{2}(k+1) w_{1}(k+1)\right) W_{1}(k)\right. \\
& \left.+\left(v_{1}(k) w_{2}(k)+v_{1}(k+1) w_{2}(k+1)\right) W_{2}(k)\right]+B_{2}
\end{aligned}
$$

with

$$
\begin{aligned}
B_{2}:= & a_{N}\left(v_{1}(N+1) w_{1}(N+1)-\left(v_{1} \cdot \mathbf{s} w_{1}\right)(N+1)\right)\left(v_{2} \cdot \mathbf{s} w_{2}\right)(2 N) \\
& +a_{N}\left(\left(v_{2} \cdot \mathbf{s} w_{2}\right)(N+1)-v_{2}(N+1) w_{2}(N+1)\right)\left(v_{1} \cdot \mathbf{s} w_{1}\right)(2 N)
\end{aligned}
$$

Multiply $E_{1}$ by $(\lambda-\mu)$ and use the recurrence relation (15) to express $(\lambda-\mu) v_{2}(k) w_{1}(k)$, $(\lambda-\mu) v_{2}(k+1) w_{1}(k+1),(\lambda-\mu) v_{1}(k) w_{2}(k)$, and $(\lambda-\mu) v_{1}(k+1) w_{2}(k+1)$ in terms of the Wronskians $W_{1}$ and $W_{2}$ to get

$$
\begin{aligned}
(\lambda-\mu) E_{1}= & \sum_{k=1}^{N}\left[a_{k} a_{k+1}\left(W_{1}(k) W_{2}(k+1)+W_{1}(k+1) W_{2}(k)\right)-a_{k-1} a_{k}\left(W_{1}(k-1) W_{2}(k)\right.\right. \\
& \left.\left.+W_{1}(k) W_{2}(k-1)\right)\right]+(\lambda-\mu) B_{2} .
\end{aligned}
$$

The sum on the right-hand side of the latter identity is a telescoping sum and equals the term $a_{1} a_{N} V$ with $V$ defined in equation (102). In a straightforward way, one sees that $\frac{(\lambda-\mu)}{a_{1} a_{N}}\left(B_{1}+B_{2}\right)$ equals the expression $B$ defined by equation (103), hence formula (101) is established.

Corollary 6.2. For any $\lambda, \mu \in \mathbb{C}$,

$$
\left\{\Delta_{\lambda}, \Delta_{\mu}\right\}_{J}=0
$$

Proof. By formula (76) for the gradient of $\Delta_{\lambda}$,

$$
\left\{\Delta_{\lambda}, \Delta_{\mu}\right\}_{J}=\left\langle\nabla_{b, a} \Delta_{\lambda}, J \nabla_{b, a} \Delta_{\mu}\right\rangle
$$

is a linear combination of terms of the form $\left\langle v_{1} \cdot \mathbf{s} w_{1}, J\left(v_{2} \cdot \mathbf{s} w_{2}\right)\right\rangle$ for pairs of fundamental solutions $v_{1}, w_{1}$ and $v_{2}, w_{2}$ of equation (11) for $\mu$ and $\lambda$, respectively. In view of equations (77) and (78), $\nabla_{b} \Delta_{\lambda}$ and $\nabla_{a} \Delta_{\lambda}$ are both $N$-periodic. In the case $\lambda \neq \mu$, we use Lemma 6.1 and 
note that the boundary terms (102) and (103) in Lemma 6.1 vanish, hence $\left\{\Delta_{\lambda}, \Delta_{\mu}\right\}_{J}=0$. In the case $\lambda=\mu$, identity (104) follows from the skew symmetry of $\{\cdot, \cdot\}_{J}$.

Corollary 6.3. Let $1 \leq k \leq 2 N$ and $\lambda \in \mathbb{C}$. On the open subset of $\mathcal{M}$ where $\lambda_{k}$ is a simple eigenvalue of $Q(b, a)$, one has

$$
\left\{\lambda_{k}, \Delta_{\lambda}\right\}_{J}=0
$$

Proof. Using formula (88) for $\nabla_{b, a} \lambda_{k}$, we conclude from Corollary 6.2 that

$$
\left\{\lambda_{k}, \Delta_{\lambda}\right\}_{J}=-\left.\frac{1}{\dot{\Delta}_{\lambda_{k}}}\left\{\Delta_{\mu}, \Delta_{\lambda}\right\}_{J}\right|_{\mu=\lambda_{k}}=0
$$

Corollary 6.4. Let $\mu_{n}$ be the $n$th Dirichlet eigenvalue of $L(b, a)$ and $\lambda \neq \mu_{n}$ a real number. Then

$$
\begin{aligned}
\left(\lambda-\mu_{n}\right)\left\langle y_{1}^{2}\left(\mu_{n}\right), J Y_{1}^{2}(\lambda)\right\rangle & =\left(a_{N} \frac{y_{1}(N+1, \lambda)}{y_{2}\left(N+1, \mu_{n}\right)}\right)^{2} \\
\left(\lambda-\mu_{n}\right)\left\langle Y_{1}^{2}\left(\mu_{n}\right), J Y_{1}(\lambda) \cdot \mathbf{s} Y_{2}(\lambda)\right\rangle & =a_{N}^{2} \frac{y_{1}(N+1, \lambda) y_{2}(N+1, \lambda)}{y_{2}\left(N+1, \mu_{n}\right)^{2}}, \\
\left(\lambda-\mu_{n}\right)\left\langle y_{1}^{2}\left(\mu_{n}\right), J Y_{2}^{2}(\lambda)\right\rangle & =a_{N}^{2}\left(\left(\frac{y_{2}(N+1, \lambda)}{y_{2}\left(N+1, \mu_{n}\right)}\right)^{2}-1\right) .
\end{aligned}
$$

Proof. The three stated identities follow from Lemma 6.1, using that $y_{1}\left(N+1, \mu_{n}\right)=0$, $y_{1}\left(2, \mu_{n}\right)=-a_{N} / a_{1}$, and, by the Wronskian identity (26), $y_{1}\left(N, \mu_{n}\right) \cdot y_{2}\left(N+1, \mu_{n}\right)=1$.

Corollary 6.5. Let $\mu_{n}$ be the $n$th Dirichlet eigenvalue of $L(b, a)$ and $\lambda \neq \mu_{n}$ a real number. Then

$$
\left\{\mu_{n}, \Delta_{\lambda}\right\}_{J}=\frac{Y_{1}(N+1, \lambda)}{\dot{Y}_{1}\left(N+1, \mu_{n}\right)} \frac{\sqrt[*]{\Delta_{\mu_{n}}^{2}-4}}{\lambda-\mu_{n}} .
$$


Proof. By equation (90), combined with equation (26), we get

$$
\left\{\mu_{n}, \Delta_{\lambda}\right\}_{J}=\frac{y_{2}\left(N+1, \mu_{n}\right)}{a_{N} \dot{Y}_{1}\left(N+1, \mu_{n}\right)}\left\langle y_{1}^{2}\left(\mu_{n}\right), J \nabla_{b, a} \Delta_{\lambda}\right\rangle
$$

Substituting formula (76) for $J \nabla_{b, a} \Delta_{\lambda}$, we obtain

$$
\begin{aligned}
\left\langle y_{1}^{2}\left(\mu_{n}\right), J \nabla_{b, a} \Delta_{\lambda}\right\rangle= & -\frac{1}{a_{N}} y_{2}(N, \lambda)\left\langle y_{1}^{2}\left(\mu_{n}\right), J Y_{1}^{2}(\lambda)\right\rangle \\
& -\frac{1}{a_{N}}\left(y_{2}(N+1, \lambda)-y_{1}(N, \lambda)\right)\left\langle y_{1}^{2}\left(\mu_{n}\right), J_{Y_{1}}(\lambda) \cdot \mathbf{s} Y_{2}(\lambda)\right\rangle \\
& +\frac{1}{a_{N}} Y_{1}(N+1, \lambda)\left\langle y_{1}^{2}\left(\mu_{n}\right), J Y_{2}^{2}(\lambda)\right\rangle .
\end{aligned}
$$

To evaluate the right side of equation (110), we apply Corollary 6.4 and get

$$
\begin{aligned}
\frac{-\mu_{n}}{a_{1} a_{N}}\left\langle y_{1}^{2}\left(\mu_{n}\right), J \nabla_{b, a} \Delta_{\lambda}\right\rangle= & \frac{1}{a_{1} y_{2}\left(N+1, \mu_{n}\right)^{2}}\left(-y_{2}(N, \lambda) y_{1}(N+1, \lambda)^{2}\right. \\
& -\left(y_{2}(N+1, \lambda)-y_{1}(N, \lambda)\right) y_{1}(N+1, \lambda) y_{2}(N+1, \lambda) \\
& \left.+y_{1}(N+1, \lambda) y_{2}(N+1, \lambda)^{2}\right)-\frac{y_{1}(N+1, \lambda)}{a_{1}} .
\end{aligned}
$$

Using the Wronskian identity (14), the sum of the terms in the square bracket of the latter expression simplifies, and one obtains

$$
\begin{aligned}
\frac{\lambda-\mu_{n}}{a_{1} a_{N}}\left\langle y_{1}^{2}\left(\mu_{n}\right), J \nabla_{b, a} \Delta_{\lambda}\right\rangle & =\frac{y_{1}(N+1, \lambda)}{a_{1} y_{2}\left(N+1, \mu_{n}\right)^{2}}-\frac{y_{1}(N+1, \lambda)}{a_{1}} \\
& =\frac{y_{1}(N+1, \lambda)}{a_{1}}\left(y_{1}\left(N, \mu_{n}\right)^{2}-1\right),
\end{aligned}
$$

where for the latter equality, we again used equation (14). Substituting equation (111) into equation (109), we get

$$
\begin{aligned}
\frac{\lambda-\mu_{n}}{a_{1} a_{N}}\left\{\mu_{n}, \Delta_{\lambda}\right\}_{J} & =\frac{y_{2}\left(N+1, \mu_{n}\right) y_{1}(N+1, \lambda)}{a_{1} a_{N} \dot{Y}_{1}\left(N+1, \mu_{n}\right)}\left(y_{1}\left(N, \mu_{n}\right)^{2}-1\right) \\
& =\frac{y_{1}(N+1, \lambda)}{a_{1} a_{N} \dot{y}_{1}\left(N+1, \mu_{n}\right)} \sqrt[*]{\Delta_{\mu_{n}}^{2}-4}
\end{aligned}
$$

where we used that, by the definition of the starred square root (29), 


$$
\sqrt[*]{\Delta_{\mu_{n}}^{2}-4}=y_{1}\left(N, \mu_{n}\right)-y_{2}\left(N+1, \mu_{n}\right)=y_{2}\left(N+1, \mu_{n}\right)\left(y_{1}\left(N, \mu_{n}\right)^{2}-1\right) .
$$

This proves equation (108).

Proposition 6.6. For any $\lambda \in \mathbb{R}, 1 \leq n \leq N-1$, and $(b, a) \in \mathcal{M} \backslash D_{n}$,

$$
\left\{\theta_{n}, \Delta_{\lambda}\right\}_{J}=\psi_{n}(\lambda)
$$

Proof. Recall that $\theta_{n}=\sum_{k=1}^{N-1} \beta_{k}^{n}(\bmod 2 \pi)$ with $\beta_{k}^{n}$ given by equations (91)-(92). To compute $\left\{\beta_{k}^{n}, \Delta_{\lambda}\right\}_{J}$, we first consider the case where $(b, a) \notin \bigcup_{k=1}^{N-1} D_{k}$ and $\lambda_{2 k}<\mu_{k}<\lambda_{2 k+1}$ for any $1 \leq k \leq N-1$. Then $\lambda_{2 k}$ and $\mu_{k}^{*}$ are smooth near $(b, a)$ and, by Leibniz's rule, we get

$$
\begin{aligned}
\left\{\beta_{k}^{n}, \Delta_{\lambda}\right\}_{J}= & \left(\int_{\lambda_{2 k}}^{\mu_{k}^{*}}\left\{\frac{\psi_{n}(\mu)}{\sqrt{\Delta_{\mu}^{2}-4}}, \Delta_{\lambda}\right\}_{J} d \mu\right. \\
& \left.+\frac{\psi_{n}\left(\mu_{k}\right)}{\sqrt[*]{\Delta_{\mu_{k}}^{2}-4}}\left\{\mu_{k}, \Delta_{\lambda}\right\}_{J}-\frac{\psi_{n}\left(\lambda_{2 k}\right)}{\sqrt[*]{\Delta_{\lambda_{2 k}}^{2}-4}}\left\{\lambda_{2 k}, \Delta_{\lambda}\right\}_{J}\right) .
\end{aligned}
$$

By Corollary 6.3, $\left\{\lambda_{2 k}, \Delta_{\lambda}\right\}_{J}=0$. Moreover, as the gradient $\nabla_{b, a} \frac{\psi_{n}(\mu)}{\sqrt{\Delta_{\mu}^{2}-4}}$ is orthogonal to $T_{b, a}$ Iso $(b, a)$ and $J \nabla_{b, a} \Delta_{\lambda} \in T_{b, a}$ Iso $(b, a)$, it follows that the Poisson bracket $\left\{\frac{\psi_{n}(\mu)}{\sqrt{\Delta_{\mu}^{2}-4}}, \Delta_{\lambda}\right\}_{J}$ vanishes for any $\mu$ in the isolating neighborhood $U_{n}$ of $G_{n}$. Hence

$$
\left\{\beta_{k}^{n}, \Delta_{\lambda}\right\}_{J}=\frac{\psi_{n}\left(\mu_{k}\right)}{\sqrt[*]{\Delta_{\mu_{k}}^{2}-4}}\left\{\mu_{k}, \Delta_{\lambda}\right\}_{J}
$$

By equation (108), we then obtain

$$
\left\{\theta_{n}, \Delta_{\lambda}\right\}_{J}=\sum_{k=1}^{N-1} \frac{\psi_{n}\left(\mu_{k}\right)}{\dot{y}_{1}\left(N+1, \mu_{k}\right)} \frac{y_{1}(N+1, \lambda)}{\lambda-\mu_{k}}=\psi_{n}(\lambda),
$$

where for the latter equality, we used that $\sum_{k=1}^{N-1} \frac{\psi_{n}\left(\mu_{k}\right)}{\dot{y}_{1}\left(N+1, \mu_{k}\right)} \frac{y_{1}(N+1, \lambda)}{\lambda-\mu_{k}}$ and $\psi_{n}(\lambda)$ are both polynomials in $\lambda$ of degree at most $N-2$ which agree at the $N-1$ points $\left(\mu_{k}\right)_{1 \leq k \leq N-1}$.

In the general case, where $(b, a) \in \mathcal{M} \backslash D_{n}$ and the Dirichlet eigenvalues are arbitrary, $\lambda_{2 k} \leq \mu_{k} \leq \lambda_{2 k+1}$ for any $1 \leq k \leq N-1$, the claimed result follows from the case treated above by continuity. 
Proposition 6.7. Let $1 \leq n, m, k, l \leq N-1$ and let $(b, a) \in \mathcal{M}$ with $\lambda_{2 i}(b, a)=\mu_{i}(b, a)$ for $i=k, l$. Then

$$
\left\{\beta_{k}^{n}, \beta_{l}^{m}\right\}_{J}=0
$$

Proof. In view of Proposition 5.4, this amounts to showing that the scalar product $\left\langle\left(g_{k} \cdot \mathbf{s}\right.\right.$ $\left.\left.h_{k}\right), J\left(g_{l} \cdot \mathbf{s} h_{l}\right)\right\rangle$ vanishes. For $k=l$, this follows from the skew symmetry of the Poisson bracket, hence we can assume that $k \neq l$. We apply Lemma 6.1 with $v_{1}:=g_{k}, w_{1}:=h_{k}, v_{2}:=$ $h_{l}$, and $w_{2}:=g_{l}$, which implies that $W_{1}=W\left(g_{k}, g_{l}\right)$ and $W_{2}=W\left(h_{k}, h_{l}\right)$. Since $g_{k}(1), g_{l}(1)$, $g_{k}(N+1)$, and $g_{l}(N+1)$ all vanish, we conclude that $W_{1}(N)=W_{1}(0)=0$ and $\left(S W_{1}\right)(N)=$ $\left(S W_{1}\right)(0)=0$, hence the expressions $V$ and $E$, defined in equations (102) and (103) vanish. This proves the claim.

\section{Canonical Relations}

In this section, we complete the proof of Theorem 1.1 and Corollary 1.2. In particular, we show that the variables $\left(I_{n}\right)_{1 \leq n \leq N-1},\left(\theta_{n}\right)_{1 \leq n \leq N-1}$ satisfy the canonical relations stated in Theorem 1.1.

Using the results of the preceding sections, we can now compute the Poisson brackets among the action and angle variables introduced in Section 3.

Theorem 7.1. The action-angle variables $\left(I_{n}\right)_{1 \leq n \leq N-1}$ and $\left(\theta_{n}\right)_{1 \leq n \leq N-1}$ satisfy the following canonical relations for $1 \leq n, m \leq N-1$ :

(i) on $\mathcal{M}$,

$$
\left\{I_{n}, I_{m}\right\}_{J}=0
$$

(ii) on $\mathcal{M} \backslash D_{n}$,

$$
\left\{\theta_{n}, I_{m}\right\}_{J}=-\left\{I_{m}, \theta_{n}\right\}_{J}=-\delta_{n m}
$$

Proof. Recall that $\frac{d}{d t} \operatorname{arcosh}(t)=\left(t^{2}-1\right)^{-\frac{1}{2}}$. Hence for any $(b, a) \in \mathcal{M}$,

$$
I_{n}=\frac{1}{2 \pi} \int_{\Gamma_{n}} \lambda \frac{d}{d \lambda} \operatorname{arcosh}\left|\frac{\Delta_{\lambda}}{2}\right| d \lambda
$$


and therefore

$$
\nabla_{b, a} I_{n}=\frac{1}{2 \pi} \int_{\Gamma_{n}} \lambda \frac{d}{d \lambda} \frac{\nabla_{b, a} \Delta_{\lambda}}{\sqrt[c]{\Delta_{\lambda}^{2}-4}} d \lambda
$$

Integrating by parts we get

$$
\nabla_{b, a} I_{n}=-\frac{1}{2 \pi} \int_{\Gamma_{n}} \frac{\nabla_{b, a} \Delta_{\lambda}}{\sqrt[c]{\Delta_{\lambda}^{2}-4}} d \lambda
$$

As $\left\{\Delta_{\lambda}, \Delta_{\mu}\right\}_{J}=0$ for all $\lambda, \mu \in \mathbb{C}$ by Corollary 6.2 , it follows that $\left\{I_{n}, I_{m}\right\}_{J}=0$ on $\mathcal{M}$ for any $1 \leq n, m \leq N-1$.

To prove equation (114), use equation (115) and then Proposition 6.6 to get

$$
\left\{\theta_{n}, I_{m}\right\}_{J}=-\frac{1}{2 \pi} \int_{\Gamma_{m}} \frac{\left\{\theta_{n}, \Delta_{\lambda}\right\}_{J}}{\sqrt[c]{\Delta_{\lambda}^{2}-4}} d \lambda=-\frac{1}{2 \pi} \int_{\Gamma_{m}} \frac{\psi_{n}(\lambda)}{\sqrt[c]{\Delta_{\lambda}^{2}-4}} d \lambda=-\delta_{n m}
$$

by the normalizing condition (36) of $\psi_{n}$.

To prove that the angles $\left(\theta_{n}\right)_{1 \leq n \leq N-1}$ pairwise Poisson commute, we need the following lemma. We denote by $K=K(b, a)$ the index set of the open gaps, that is,

$$
K(b, a)=\left\{1 \leq n \leq N-1: \gamma_{n}(b, a)>0\right\} .
$$

Lemma 7.2. At every point $(b, a)$ in $\mathcal{M}$, the set of vectors

(i)

$$
\left(\left(\nabla_{b, a} I_{n}\right)_{n \in K}, \nabla_{b, a} C_{1}, \nabla_{b, a} C_{2}\right)
$$

and

$$
\left(J \nabla_{b, a} I_{n}\right)_{n \in K}
$$

are both linearly independent.

Proof. The claimed statements follow from the orthogonality relations stated in Theorem 7.1: Let $(b, a) \in \mathcal{M}$ and suppose that for some real coefficients $\left(r_{n}\right)_{n \in K} \subseteq \mathbb{R}$ and $s_{1}, s_{2} \in \mathbb{R}$ we have

$$
\sum_{n \in K} r_{n} \nabla_{b, a} I_{n}+s_{1} \nabla_{b, a} C_{1}+s_{2} \nabla C_{2}=0 .
$$


For any $m \in K$, take the scalar product of this identity with $J \nabla_{b, a} \theta_{m}$. Using that $\left\{I_{n}, \theta_{m}\right\}_{J}=$ $\delta_{n m}$ and that $C_{1}$ and $C_{2}$ are Casimir functions of $\{\cdot, \cdot\}_{J}$, one obtains

$$
0=\sum_{n \in K} r_{n}\left\{I_{n}, \theta_{m}\right\}_{J}=\sum_{n \in K} r_{n} \delta_{n m}=r_{m}
$$

Thus $r_{m}=0$ for all $m \in K$, and it follows that $s_{1} \nabla_{b, a} C_{1}+s_{2} \nabla_{b, a} C_{2}=0$. By equations (8) and (9), $\nabla_{b, a} C_{1}$ and $\nabla_{b, a} C_{2}$ are linearly independent, hence $s_{1}=s_{2}=0$. This proves (i). The proof of (i) also shows that (ii) holds.

Theorem 7.3. In addition to the canonical relations stated in Theorem 7.1, the angle variables $\left(\theta_{n}\right)_{1 \leq n \leq N-1}$ satisfy for any $1 \leq n, m \leq N-1$ on $\mathcal{M} \backslash\left(D_{n} \cup D_{m}\right)$

$$
\left\{\theta_{n}, \theta_{m}\right\}_{J}=0
$$

Proof. Let $1 \leq n, m \leq N-1$. By continuity, it suffices to prove identity (116) for $(b, a) \in$ $\mathcal{M} \backslash\left(\bigcup_{l=1}^{N-1} D_{l}\right)$. Let $(b, a)$ be an arbitrary element in $\mathcal{M} \backslash\left(\bigcup_{l=1}^{N-1} D_{l}\right)$. Recall that Iso $(b, a)$ denotes the set of all elements $\left(b^{\prime}, a^{\prime}\right)$ in $\mathcal{M}$ with $\operatorname{spec}\left(Q_{b^{\prime}, a^{\prime}}\right)=\operatorname{spec}\left(Q_{b, a}\right)$,

$$
\text { Iso }(b, a)=\left\{\left(b^{\prime}, a^{\prime}\right) \in \mathcal{M}: \Delta\left(\cdot, b^{\prime}, a^{\prime}\right)=\Delta(\cdot, b, a)\right\} \text {. }
$$

Then Iso $(b, a)$ is a torus contained in $\mathcal{M} \backslash\left(\bigcup_{l=1}^{N-1} D_{l}\right)$, and as all eigenvalues of $Q(b, a)$ are simple, its dimension is $N-1$. By Lemma 7.2, at any point $\left(b^{\prime}, a^{\prime}\right) \in \operatorname{Iso}(b, a)$, the vectors $\left(J \nabla_{b^{\prime}, a^{\prime}} I_{k}\right)_{1 \leq k \leq N-1}$ are linearly independent. Using formula (115) for the gradient of $I_{k}$, one sees that, by Corollary 6.2 , for any $\mu \in \mathbb{R}, 1 \leq k \leq N-1$,

$$
\left\langle\nabla_{b^{\prime}, a^{\prime}} \Delta_{\mu}, J \nabla_{b^{\prime}, a^{\prime}} I_{k}\right\rangle=-\frac{1}{2 \pi} \int_{\Gamma_{n}} \frac{\left\{\Delta_{\mu}, \Delta_{\lambda}\right\}_{J}}{\sqrt[d]{\Delta_{\lambda}^{2}-4}} d \lambda=0
$$

Hence for any $\left(b^{\prime}, a^{\prime}\right) \in \operatorname{Iso}(b, a)$,

$$
\left(J \nabla_{b^{\prime}, a^{\prime}} I_{k}\right)_{1 \leq k \leq N-1} \in T_{b^{\prime}, a^{\prime}} \text { Iso }(b, a),
$$

and therefore these vectors form a basis of $T_{b^{\prime}, a^{\prime}}$ Iso $(b, a)$. 
To prove identity (116), we apply the Jacobi identity

$$
\left\{F,\{G, H\}_{J}\right\}_{J}+\left\{G,\{H, F\}_{J}\right\}_{J}+\left\{H,\{F, G\}_{J}\right\}_{J}=0
$$

to the functions $I_{k}, \theta_{n}$, and $\theta_{m}$. Since by Theorem $7.1\left\{I_{k}, \theta_{n}\right\}_{J}=\delta_{k n}$, we obtain

$$
\left\{I_{k},\left\{\theta_{n}, \theta_{m}\right\}_{J}\right\}_{J}=0 \text { on } \mathcal{M} \backslash\left(\bigcup_{l=1}^{N-1} D_{l}\right) \text { for any } 1 \leq k \leq N-1
$$

It then follows by the above considerations that $\nabla_{b^{\prime}, a^{\prime}}\left\{\theta_{n}, \theta_{m}\right\}_{J}$ is orthogonal to $T_{b^{\prime}, a^{\prime}}$ Iso $(b, a)$ for all $\left(b^{\prime}, a^{\prime}\right) \in \operatorname{Iso}(b, a)$, i.e. $\left\{\theta_{n}, \theta_{m}\right\}_{J}$ is constant on $\operatorname{Iso}(b, a)$,

$$
\left\{\theta_{n}, \theta_{m}\right\}_{J}\left(b^{\prime}, a^{\prime}\right)=\left\{\theta_{n}, \theta_{m}\right\}_{J}(b, a) \quad \forall\left(b^{\prime}, a^{\prime}\right) \in \operatorname{Iso}(b, a) .
$$

By [16], Theorem 2.1, there exists a unique element $\left(b^{\prime}, a^{\prime}\right) \in \operatorname{Iso}(b, a)$ satisfying $\mu_{k}\left(b^{\prime}, a^{\prime}\right)=$ $\lambda_{2 k}(b, a)$ for all $1 \leq k \leq N-1$. The claimed identity (116) then follows from Proposition 6.7 .

Proof of Theorem 1.1 By Theorems 3.5 and 4.2, the action and angle variables introduced in Definitions 3.1 and 4.1, respectively, have the claimed analyticity properties. The canonical relations among these variables have been verified in Theorems 7.1 and 7.3, and the relations $\left\{C_{i}, I_{n}\right\}_{J}=0$ (on $\mathcal{M}$ ) and $\left\{C_{i}, \theta_{n}\right\}_{J}=0$ (on $\mathcal{M} \backslash D_{n}$ ) follow from the fact that $C_{1}$ and $C_{2}$ are Casimir functions. It remains to show that the actions Poisson commute with the Toda Hamiltonian. To this end, note that that the Hamiltonian $H$ can be written as

$$
H=\frac{1}{2} \sum_{n=1}^{N} b_{n}^{2}+\sum_{n=1}^{N} a_{n}^{2}=\frac{1}{2} \operatorname{tr}\left(L(b, a)^{2}\right)=\frac{1}{2} \sum_{j=1}^{N}\left(\lambda_{j}^{+}\right)^{2}
$$

where $\left(\lambda_{j}^{+}\right)_{1 \leq j \leq N}$ are the $N$ eigenvalues of $L(b, a)$. Recall that on the dense open subset $\mathcal{M} \backslash \cup_{k=1}^{N-1} D_{k}$ of $\mathcal{M}$, the $\lambda_{i}^{+}$'s $(1 \leq i \leq N)$ are simple eigenvalues and hence real analytic. It then follows by equation (115) that for any $1 \leq n \leq N-1$,

$$
\left\{H, I_{n}\right\}_{J}=\sum_{i=1}^{N} \lambda_{i}^{+}\left\{\lambda_{i}^{+}, I_{n}\right\}_{J}=-\sum_{i=1}^{N} \frac{\lambda_{i}^{+}}{2 \pi} \int_{\Gamma_{n}} \frac{\left\{\lambda_{i}^{+}, \Delta_{\lambda}\right\}_{J}}{\sqrt[c]{\Delta_{\lambda}^{2}-4}} d \lambda=0
$$


where for the latter identity we used Corollary 6.3. Hence for any $1 \leq n \leq N-1$,

$$
\left\{H, I_{n}\right\}_{J}=0 \quad \text { on } \mathcal{M} \backslash \cup_{k=1}^{N-1} D_{k} .
$$

By continuity, it then follows that $\left\{H, I_{n}\right\}_{J}=0$ everywhere on $\mathcal{M}$.

Proof of Corollary 1.2 Since for any $\beta \in \mathbb{R}$ and $\alpha>0$, the symplectic leaf $\mathcal{M}_{\beta, \alpha}$ is a submanifold of $\mathcal{N}$ of dimension $2(N-1)$, there are at most $N-1$ independent integrals in involution on $\mathcal{M}_{\beta, \alpha}$. For any given $(b, a) \in \mathcal{M}_{\beta, \alpha}$, let $\pi_{\beta, \alpha}$ denote the orthogonal projection $T_{b, a} \mathcal{M} \rightarrow T_{b, a} \mathcal{M}_{\beta, \alpha}$. Then the gradient of the restriction $\left.I_{n}\right|_{\mathcal{M}_{\beta, \alpha}}$ of $I_{n}$ to $\mathcal{M}_{\beta, \alpha}(1 \leq n \leq N-1)$ is given by $\pi_{\beta, \alpha} \nabla_{b, a} I_{n}$. By Lemma 7.2, the vectors $\left(\pi_{\beta, \alpha} \nabla_{b, a} I_{n}\right)_{n \in K}$ are linearly independent. As $\mathcal{M}_{\beta, \alpha} \backslash \cup_{k=1}^{N-1} D_{k}$ is dense in $\mathcal{M}_{\beta, \alpha}$, it then follows that $\left(\left.I_{n}\right|_{\mathcal{M}_{\beta, \alpha}}\right)_{1 \leq n \leq N-1}$ are functionally independent. Finally, as $C_{1}$ and $C_{2}$ are Casimir functions of $\{\cdot, \cdot\}_{J}$, it follows that for any $(b, a) \in \mathcal{M}_{\beta, \alpha}$,

$$
\begin{aligned}
& \left\{\left.\left.I_{n}\right|_{\mathcal{M}_{\beta, \alpha}} I_{m}\right|_{\mathcal{M}_{\beta, \alpha}}\right\}_{J}(b, a)=\left\langle\pi_{\beta, \alpha} \nabla_{b, a} I_{n}, \pi_{\beta, \alpha} J \nabla_{b, a} I_{m}\right\rangle \\
& =\left\langle\nabla_{b, a} I_{n}, J \nabla_{b, a} I_{m}\right\rangle=\left\{I_{n}, I_{m}\right\}_{J}=0,
\end{aligned}
$$

that is, the restrictions $\left.I_{n}\right|_{\mathcal{M}_{\beta, \alpha}}$ of $I_{n}(1 \leq n \leq N-1)$ are in involution.

\section{A Appendix Proof of Lemma 3.7}

In this Appendix, we prove Lemma 3.7. It turns out that the proof in ([1], pp. 601-602) of the special case where the parameter $\alpha$ in equation (1) equals 1 can be adapted for arbitrary values.

Proof of Lemma 3.7 Let $(b, a)$ be an arbitrary element in $\mathcal{M}$ and $1 \leq n \leq N-1$. First note that $I_{n}=\frac{1}{\pi} \int_{\lambda_{2 n}}^{\lambda_{2 n+1}} \operatorname{arcosh}\left|\frac{1}{2} \Delta(\lambda)\right| d \lambda$ and use $\frac{d}{d t} \operatorname{arcosh}(t)=\frac{1}{\sqrt{t^{2}-1}}$ to obtain

$$
I_{n}=\frac{1}{\pi} \int_{\lambda_{2 n}}^{\lambda_{2 n+1}} \int_{1}^{|\Delta(\lambda)| / 2} \frac{1}{\sqrt{t^{2}-1}} d t d \lambda
$$

Since the integrand of the inner integral is nonincreasing, we estimate it by its value at $\frac{|\Delta(\lambda)|}{2}$. This leads to

$$
I_{n} \geq \frac{1}{\pi} \int_{\lambda_{2 n}}^{\lambda_{2 n+1}} \frac{\sqrt{|\Delta(\lambda)|-2}}{\sqrt{|\Delta(\lambda)|+2}} d \lambda
$$


We will show that for $\lambda_{2 n} \leq \lambda \leq \lambda_{2 n+1}$,

$$
\frac{\sqrt{|\Delta(\lambda)|-2}}{\sqrt{|\Delta(\lambda)|+2}} \geq \frac{\sqrt{\lambda-\lambda_{2 n}} \sqrt{\lambda_{2 n+1}-\lambda}}{\lambda_{2 N}-\lambda_{1}} .
$$

We then substitute equation (A.2) into the integral (A.1) and split the integration interval into two equal parts,

$$
I_{n} \geq \frac{2}{\pi} \frac{1}{\lambda_{2 N}-\lambda_{1}} \int_{\lambda_{2 n}}^{\tau_{n}} \frac{\sqrt{\lambda-\lambda_{2 n}} \sqrt{\lambda_{2 n+1}-\lambda}}{\lambda_{2 N}-\lambda_{1}} d \lambda,
$$

where $\tau_{n}=\left(\lambda_{2 n}+\lambda_{2 n+1}\right) / 2$. For $\lambda_{2 n} \leq \lambda \leq \tau_{n}$, we estimate the quantity $\lambda_{2 n+1}-\lambda$ from below by $\gamma_{n} / 2$, yielding

$$
I_{n} \geq \frac{2}{\pi} \frac{1}{\lambda_{2 N}-\lambda_{1}} \int_{\lambda_{2 n}}^{\tau_{n}} \sqrt{\frac{\gamma_{n}}{2}} \sqrt{\lambda-\lambda_{2 n}} d \lambda=\frac{1}{3 \pi\left(\lambda_{2 N}-\lambda_{1}\right)} \gamma_{n}^{2} .
$$

It remains to verify equation (A.2). If $\lambda_{2 n}$ and $\lambda_{2 n+1}$ are periodic eigenvalues, we have $\Delta(\lambda) \geq 2$ for $\lambda_{2 n} \leq \lambda \leq \lambda_{2 n+1}$, i.e. $|\Delta(\lambda)|=\Delta(\lambda)$. In order to make writing easier, let us assume that $N$ is even-the case where $N$ is odd is treated in the same way. Then by equation (19), $\lambda_{1}$ and $\lambda_{2 N}$ are periodic eigenvalues of $L$ and thus for any $\lambda_{2 n} \leq \lambda \leq \lambda_{2 n+1}$, the left side of equation (A.2) can be estimated by

$$
\frac{\sqrt{\Delta(\lambda)-2}}{\sqrt{\Delta(\lambda)+2}}=\sqrt{\frac{\left(\lambda-\lambda_{2 n}\right)\left(\lambda-\lambda_{2 n+1}\right)}{\left(\lambda-\lambda_{2}\right)\left(\lambda-\lambda_{2 N-1}\right)}} \cdot R \geq \frac{\sqrt{\lambda-\lambda_{2 n}} \sqrt{\lambda_{2 n+1}-\lambda}}{\lambda_{2 N}-\lambda_{1}} \cdot R
$$

where

$$
R \equiv R(\lambda)=\sqrt{\frac{\lambda-\lambda_{1}}{\lambda-\lambda_{3}} \cdots \frac{\lambda-\lambda_{2 n-3}}{\lambda-\lambda_{2 n-1}} \frac{\lambda_{2 n+4}-\lambda}{\lambda_{2 n+2}-\lambda} \cdots \frac{\lambda_{2 N}-\lambda}{\lambda_{2 N-2}-\lambda}} .
$$

As each of the the fractions under the square root in equation (A.3) can be estimated from below by 1 , for any $\lambda_{2 n} \leq \lambda \leq \lambda_{2 n+1}$ it follows that $R(\lambda) \geq 1$ on $\left[\lambda_{2 n}, \lambda_{2 n+1}\right]$, leading to the claimed estimate (A.2).

\section{B Appendix Proof of Theorem 3.9}

In this Appendix, we prove Theorem 3.9 using estimates derived in [1]. Let $(b, a)$ be in $\mathcal{M}_{\beta, \alpha}$ with $\beta \in \mathbb{R}$ and $\alpha>0$ arbitrary.

To show Theorem 3.9, we need the following proposition. 
Proposition B.1. For any $(b, a) \in \mathcal{M}_{\beta, \alpha}$ with $\beta \in \mathbb{R}, \alpha>0$ arbitrary and any $1 \leq n \leq N$,

$$
\lambda_{2 n}(b, a)-\lambda_{2 n-1}(b, a) \leq \frac{2 \pi \alpha}{N}
$$

Before proving Proposition B.1 we show how to use it to prove Theorem 3.9.

Proof of Theorem 3.9 We begin by adding up the inequalities (60) and get

$$
\sum_{n=1}^{N-1} \gamma_{n}^{2} \leq 3 \pi\left(\lambda_{2 N}-\lambda_{1}\right)\left(\sum_{n=1}^{N-1} I_{n}\right)
$$

Note that

$$
\lambda_{2 N}-\lambda_{1}=\sum_{n=1}^{N-1} \gamma_{n}+\sum_{n=1}^{N}\left(\lambda_{2 n}-\lambda_{2 n-1}\right)
$$

By the estimate of Proposition B.1 we get for any $(b, a) \in \mathcal{M}_{\beta, \alpha}$,

$$
\lambda_{2 N}-\lambda_{1} \leq 2 \pi \alpha+\sum_{n=1}^{N-1} \gamma_{n}
$$

which we substitute into equation (B.2) to yield

$$
\sum_{n=1}^{N-1} \gamma_{n}^{2} \leq 6 \alpha \pi^{2}\left(\sum_{n=1}^{N-1} I_{n}\right)+3 \pi\left(\sum_{n=1}^{N-1} \gamma_{n}\right)\left(\sum_{n=1}^{N-1} I_{n}\right)
$$

Using the inequality

$$
2 a b \leq \epsilon^{2} a^{2}+\frac{1}{\epsilon^{2}} b^{2} \quad(a, b \in \mathbb{R}, \epsilon>0)
$$

with $a=\sum_{n=1}^{N-1} \gamma_{n}, b=\sum_{n=1}^{N-1} I_{n}$, and $\epsilon^{2}=\frac{1}{3 \pi(N-1)}$, one gets

$$
\sum_{n=1}^{N-1} \gamma_{n}^{2} \leq 6 \pi^{2} \alpha\left(\sum_{n=1}^{N-1} I_{n}\right)+\frac{3 \pi}{2}\left(\frac{1}{3 \pi(N-1)}\left(\sum_{n=1}^{N-1} \gamma_{n}\right)^{2}+3 \pi(N-1)\left(\sum_{n=1}^{N-1} I_{n}\right)^{2}\right)
$$


As $\left(\sum_{n=1}^{N-1} \gamma_{n}\right)^{2} \leq(N-1)\left(\sum_{n=1}^{N-1} \gamma_{n}^{2}\right)$, one then concludes that

$$
\frac{1}{2} \sum_{n=1}^{N-1} \gamma_{n}^{2} \leq 6 \pi^{2} \alpha\left(\sum_{n=1}^{N-1} I_{n}\right)+\frac{9 \pi^{2}}{2}(N-1)\left(\sum_{n=1}^{N-1} I_{n}\right)^{2}
$$

which is the claimed estimate (61).

To prove Proposition B.1 we first need to make some preparations. Note that for an element of the form $(b, a)=\left(\beta 1_{N}, \alpha 1_{N}\right)$ one has, by Lemma 2.6,

$$
\begin{aligned}
\lambda_{2 n}\left(\beta 1_{N}, \alpha 1_{N}\right)-\lambda_{2 n-1}\left(\beta 1_{N}, \alpha 1_{N}\right) & =2 \alpha\left(\cos \frac{(n-1) \pi}{N}-\cos \frac{n \pi}{N}\right) \\
& =4 \alpha \sin \frac{(2 n-1) \pi}{2 N} \sin \frac{\pi}{2 N} \\
& <\frac{2 \pi \alpha}{N} .
\end{aligned}
$$

Hence to prove Proposition B.1, it suffices to show that for any $(b, a) \in \mathcal{M}_{\beta, \alpha}$ and any $1 \leq n \leq N$,

$$
\lambda_{2 n}(b, a)-\lambda_{2 n-1}(b, a) \leq \lambda_{2 n}\left(-\beta 1_{N}, \alpha 1_{N}\right)-\lambda_{2 n-1}\left(-\beta 1_{N}, \alpha 1_{N}\right)
$$

To this end, following [14] (cf. also [7]), we introduce the conformal map

$$
\delta(\lambda):=(-1)^{N} \int_{\lambda_{1}}^{\lambda} \frac{\dot{\Delta}(\mu)}{\sqrt{4-\Delta^{2}(\mu)}} d \mu
$$

where the sign of the square root is chosen such that for $\mu<\lambda_{1}, \sqrt{4-\Delta^{2}(\mu)}$ has positive imaginary part. It is defined on the upper half plane $U:=\{\operatorname{Im} z>0\}$ and its image is the spike domain

$$
\Omega(b, a):=\{x+i y: 0<x<N \pi, y>0\} \backslash \bigcup_{n=1}^{N-1} T_{n}
$$

where for $1 \leq n \leq N-1, T_{n}$ denotes the spike

$$
T_{n}:=\left\{n \pi+i t: 0<t \leq \operatorname{arcosh}\left((-1)^{N+n} \frac{\Delta\left(\dot{\lambda}_{n}\right)}{2}\right)\right\}
$$


To see that $\delta(U)=\Omega(b, a)$, note that for any $(b, a) \in \mathcal{M}$ and $\lambda \in U$. the discriminant $\Delta(\lambda)$ and the function $\delta(\lambda)$ are related by the formula

$$
\Delta(\lambda)=2(-1)^{N} \cos \delta(\lambda)
$$

To prove equation (B.6), recall that for $-1<t<1$, one has $\frac{d}{d t} \arccos t=\frac{1}{\sqrt[+]{1-t^{2}}}$. This formula remains valid for any $t$ in $\mathbb{C} \backslash((-\infty,-1] \cup[1, \infty))$. Thus

$$
\delta(\lambda)=(-1)^{N} \int_{\lambda_{1}}^{\lambda} \frac{\dot{\Delta}(\mu)}{\sqrt{4-\Delta^{2}(\mu)}} d \mu=\int_{\lambda_{1}}^{\lambda} \frac{d}{d \mu} \arccos \left((-1)^{N} \frac{\Delta(\mu)}{2}\right) .
$$

Since by equation (19) $\Delta\left(\lambda_{1}\right)=2(-1)^{N}$, we then get

$$
\delta(\lambda)=\left.\arccos \left((-1)^{N} \frac{\Delta(\mu)}{2}\right)\right|_{\lambda_{1}} ^{\lambda}=\arccos \left((-1)^{N} \frac{\Delta(\lambda)}{2}\right)
$$

leading to formula (B.6) and the claimed statement that $\delta(U)=\Omega(b, a)$.

The map $\delta$ can be extended continuously to the closure $\{\operatorname{Im} z \geq 0\}$ of the upper half plane. This extension, again denoted by $\delta$, is 2-1 over each nontrivial spike $T_{n}$ and 1-1 otherwise. Since the $n$th spike $T_{n}$ is the image under $\delta$ of the $n$th gap $\left(\lambda_{2 n}, \lambda_{2 n+1}\right)$, all spikes are empty if and only if all gaps are collapsed. By Lemma 2.6, all gaps are collapsed for $(b, a)=\left(-\beta 1_{N}, \alpha 1_{N}\right)$, hence $\Omega(b, a) \subset \Omega\left(-\beta 1_{N}, \alpha 1_{N}\right)$ for any $(b, a) \in \mathcal{M}_{\beta, \alpha}$.

Note that

$$
\lambda_{2 n}(b, a)-\lambda_{2 n-1}(b, a)=\delta^{-1}(n \pi-)-\delta^{-1}((n-1) \pi+)=\int_{-\infty}^{\infty} u^{(n)}(\delta(\lambda)) d \lambda,
$$

where $u^{(n)}: \Omega(b, a) \rightarrow \mathbb{R}, z \mapsto u^{(n)}(z ; b, a)$ is the harmonic measure of the open subset $((n-$ 1) $\pi, n \pi)$ of $\partial \Omega(b, a)$ (see e.g. [6] for the notion of the harmonic measure).

We need two lemmas from complex and harmonic analyses, respectively.

Lemma B.1. For $(b, a)=\left(-\beta 1_{N}, \alpha 1_{N}\right)$ with $\beta \in \mathbb{R}, \alpha>0$ arbitrary, the map $\delta(\lambda)$ defined by equation (B.5) is given by

$$
\delta(\lambda)=N \arccos \left(-\frac{\lambda+\beta}{2 \alpha}\right)
$$


For arbitrary $(b, a)$ in $\mathcal{M}_{\beta, \alpha}$ and $\xi \in \mathbb{R}$, the following asymptotic estimate holds as $\eta \rightarrow \infty$ :

$$
\delta(\xi+i \eta)=N \arccos \left(-\frac{\xi+i \eta+\beta}{2 \alpha}\right)+O\left(\eta^{-2}\right)
$$

locally uniformly in $\xi$.

Proof of Lemma B.1 In view of formulas (51) and (52) for the fundamental solutions $y_{1}$ and $y_{2}$ for $(b, a)=\left(-\beta 1_{N}, \alpha 1_{N}\right)$, the discriminant $\Delta(\lambda)=\Delta\left(\lambda,-\beta 1_{N}, \alpha 1_{N}\right)$ is given by

$$
\begin{aligned}
\Delta(\lambda) & =y_{1}(N, \lambda)+y_{2}(N+1, \lambda) \\
& =-\frac{\sin (\rho(N-1))}{\sin \rho}+\frac{\sin (\rho(N+1))}{\sin \rho} \\
& =2 \cos (\rho N),
\end{aligned}
$$

where $\pi<\rho<2 \pi$ is determined by $\cos \rho=\frac{\lambda+\beta}{2 \alpha}$. Hence

$$
\Delta(\lambda)=2 T_{N}\left(\frac{\lambda+\beta}{2 \alpha}\right)
$$

where for any $z \in U$,

$$
T_{N}(z)=\cos (N \arccos z)
$$

Actually, $T_{N}(z)$ is a polynomial in $z$ of degree $N$, referred to as Chebychev polynomial of the first kind. Substituting equation (B.11) into equation (B.7), we obtain

$$
\delta(\lambda)=\arccos \left((-1)^{N} \frac{\Delta(\lambda)}{2}\right)=\arccos \left((-1)^{N} T_{N}\left(\frac{\lambda+\beta}{2 \alpha}\right)\right) .
$$

The claimed identity (B.9) now follows from the elementary symmetry

$$
T_{N}(z)=(-1)^{N} T_{N}(-z) \quad \forall z \in \mathbb{C} .
$$

Now let $(b, a) \in \mathcal{M}_{\beta, \alpha}$. The asymptotic estimate (B.10) follows by comparing the polynomials $\Delta(\lambda)$ correponding to $(b, a)$ and the one corresponding to $\left(-\beta 1_{N}, \alpha 1_{N}\right)$. By equation (17) (and the discussion following it), in both cases,

$$
\Delta(\lambda)=\alpha^{-N} \lambda^{N}\left(1+N \beta \lambda^{-1}+O\left(\lambda^{-2}\right)\right) \quad \text { as } \quad|\lambda| \rightarrow \infty .
$$


This implies that

$$
\Delta_{b, a}(\lambda)=\Delta_{-\beta 1_{N}, \alpha 1_{N}}(\lambda) \cdot\left(1+O\left(\lambda^{-2}\right)\right),
$$

hence by equations (B.9) and (B.11),

$$
\begin{aligned}
\delta_{b, a}(\lambda) & =\arccos \left((-1)^{N} \frac{\Delta_{b, a}(\lambda)}{2}\right) \\
& =\arccos \left(\frac{(-1)^{N}}{2} \Delta_{-\beta 1_{N}, \alpha 1_{N}}(\lambda) \cdot\left(1+O\left(\lambda^{-2}\right)\right)\right) . \\
& =\arccos \left((-1)^{N} T_{N}\left(\frac{\lambda+\beta}{2 \alpha}\right) \cdot\left(1+O\left(\lambda^{-2}\right)\right)\right) \\
& =\arccos \left(T_{N}\left(-\frac{\lambda+\beta}{2 \alpha}\right) \cdot\left(1+O\left(\lambda^{-2}\right)\right)\right) .
\end{aligned}
$$

Substituting formula (B.12) for $T_{N}$, one then concludes that

$$
\begin{aligned}
\delta_{b, a}(\lambda) & =\arccos \left(\cos \left(N \arccos \left(-\frac{\lambda+\beta}{2 \alpha}\right)\right) \cdot\left(1+O\left(\lambda^{-2}\right)\right)\right) \\
& =N \arccos \left(-\frac{\lambda+\beta}{2 \alpha}\right)+O\left(\lambda^{-2}\right),
\end{aligned}
$$

where in the last step we used that $\arccos z=-i \log \left(z+i \sqrt[+]{1-z^{2}}\right)$ for any $z$ in $\mathbb{C} \backslash$ $((-\infty,-1] \cup[1, \infty))$.

Lemma B.3. Let $u: \Omega(b, a) \rightarrow \mathbb{R}$ be a bounded harmonic function such that the nontangential limit of $u(z)$ on $\partial \Omega(b, a)$ has compact support, and let $U(\lambda):=u(\delta(\lambda))$, where $\delta(\lambda)$ is the function defined by equation (B.5). Then for almost every $t \in \mathbb{R}$, the limit $U(t):=\lim _{\eta \rightarrow 0} U(t+i \eta)$ exists and is integrable, and

$$
\int_{-\infty}^{\infty} U(t) d t=\lim _{x \rightarrow \infty} 2 \pi \alpha \sinh \left(\frac{x}{N}\right) u\left(\frac{N \pi}{2}+i x\right)
$$

Proof of Lemma B.3 Again by Fatou's theorem, for a.e. $t$, the (nontangential) limit $\lim _{\eta \rightarrow 0} U(t+i \eta)$ exists, since $U$ is a bounded harmonic function on $\{\operatorname{Im}(z)>0\}$. Since $u$ is bounded on $\Omega(b, a)$ and its nontangential limit to $\partial \Omega$ has compact support, $U(t)$ is bounded and compactly supported, and thus in particular, integrable. For $\lambda=\xi+i \eta$, one 
then has the Poisson representation

$$
U(\xi+i \eta)=\frac{\eta}{\pi} \int_{-\infty}^{\infty} \frac{U(t)}{(t-\xi)^{2}+\eta^{2}}
$$

and by dominated convergence we conclude that

$$
\int_{-\infty}^{\infty} U(t) d t=\lim _{\eta \rightarrow \infty} \pi \eta U(\xi+i \eta)
$$

(In particular, the limit in the latter expression exists.) In order to compute the right-hand side of equation (B.16), let $\xi+i \eta$ be given by

$$
\xi+i \eta=\delta^{-1}\left(\frac{N \pi}{2}+i x\right)
$$

for $x$ sufficiently large. Then $U(\xi+i \eta)=u\left(\frac{N \pi}{2}+i x\right)$, and by equation (B.13), it follows that

$$
\frac{\pi}{2}+\frac{i}{N} x=\arccos \left(-\frac{(\xi+\beta)+i \eta}{2 \alpha}\right)+O\left((\xi+i \eta)^{-2}\right) \quad(x \rightarrow \infty)
$$

Taking the cosine of both sides of equation (B.17), multiplying by $-2 \alpha$, and using that $\cos \left(\frac{\pi}{2}+i t\right)=-i \sinh t$ for $t \in \mathbb{R}$, we obtain

$$
2 i \alpha \sinh \frac{x}{N}=((\xi+\beta)+i \eta)\left(1+O\left((\xi+i \eta)^{-2}\right)\right) \quad(x \rightarrow \infty) .
$$

Hence, as $x \rightarrow \infty$,

$$
\xi=O(1), \quad \eta=2 \alpha \sinh \frac{x}{N}+O(1)
$$

Substituting equation (B.18) into equation (B.16) leads to the claimed formula (B.14).

Proof of Proposition B.1 Let $(b, a) \in \mathcal{M}_{\beta, \alpha}$. Besides the harmonic measure $u^{(n)}$ of the set $E:=((n-1) \pi, n \pi) \subset \partial \Omega(b, a)$, we also consider the harmonic measure $u_{\beta, \alpha}^{(n)}$ of $E \subset$ $\partial \Omega\left(-\beta 1_{N}, \alpha 1_{N}\right)$; note that

$$
\Omega\left(-\beta 1_{N}, \alpha 1_{N}\right)=\{x+i y \mid 0<x<N \pi, y>0\},
$$

and hence $\Omega(b, a) \subseteq \Omega\left(-\beta 1_{N}, \alpha 1_{N}\right)$. According to [6], both $u^{(n)}$ and $u_{\beta, \alpha}^{(n)}$ satisfy the hypotheses of Lemma B.3. Let us recall the monotonicity property of the harmonic measures 
$u(z, E, \Omega)$ with respect to $\Omega$ (see e.g. [6]): If $\Omega_{1} \subseteq \Omega_{2}, E \subset \partial \Omega_{1} \cap \partial \Omega_{2}$, and $u\left(z, E, \Omega_{i}\right)(i=1,2)$ denotes the harmonic measure of $E \subset \partial \Omega_{i}$, then for any $z \in \Omega_{1}, u\left(z, E, \Omega_{1}\right) \leq u\left(z, E, \Omega_{2}\right)$. Apply this general principle to $\Omega_{1}:=\Omega(b, a)$ and $\Omega_{2}:=\Omega\left(-\beta 1_{N}, \alpha 1_{N}\right)$ to get

$$
u^{(n)}(x) \leq u_{\beta, \alpha}^{(n)}(x)
$$

Writing $U^{(n)}(\lambda):=u^{(n)}(\delta(\lambda))$ as well as $U_{\beta, \alpha}^{(n)}(\lambda):=u_{\beta, \alpha}^{(n)}(\delta(\lambda))$ and combining equations (B.8), (B.14), and (B.19), we conclude that

$$
\begin{aligned}
\lambda_{2 n}(b, a)-\lambda_{2 n-1}(b, a) & =\int_{-\infty}^{\infty} U^{(n)}(\lambda) d \lambda \\
& =\lim _{X \rightarrow \infty} 2 \pi \alpha \sinh \left(\frac{x}{N}\right) u^{(n)}\left(\frac{N \pi}{2}+i x\right) \\
& \leq \lim _{X \rightarrow \infty} 2 \pi \alpha \sinh \left(\frac{x}{N}\right) u_{\beta, \alpha}^{(n)}\left(\frac{N \pi}{2}+i x\right) \\
& =\int_{-\infty}^{\infty} U_{\beta, \alpha}^{(n)}(\lambda) d \lambda \\
& =\lambda_{2 n}\left(-\beta 1_{N}, \alpha 1_{N}\right)-\lambda_{2 n-1}\left(-\beta 1_{N}, \alpha 1_{N}\right) .
\end{aligned}
$$

This completes the proof of estimate (B.1) and therefore of Proposition B.1.

\section{Acknowledgments}

Supported in part by the Swiss National Science Foundation, the programme SPECT, and the European Community through the FP6 Marie Curie RTN ENIGMA (MRTN-CT-2004-5652)

\section{References}

[1] Bättig, D., A. M. Bloch, J. C. Guillot, and T. Kappeler. "On the symplectic structure of the phase space for periodic KdV, Toda, and defocusing NLS." Duke Mathematical Journal 79 (1995): 549-604.

[2] Bättig, D., B. Grébert, J. C. Guillot, and T. Kappeler. "Fibration of the phase space of the periodic Toda lattice." Journal de Math¥ematiques Pures et Appliqu¥ees 72 (1993): 553-65.

[3] Fermi, E., J. Pasta, and S. Ulam. "Studies of non linear problems." In Collected Papers of Enrico Fermi. vol. 2, 978-88. Los Alamos Rpt, LA-1940 (1955). Chicago, IL: University of Chicago Press, 1965. Theory, Methods and Applications, 2nd ed. New York: Marcel Dekker, 2000.

[4] Flaschka, H. “The Toda lattice. Existence of integrals." Physical Review, Sec. B 9, no. 1 (1974): 1924-5. 
[5] Flaschka, H., and D. McLaughlin. "Canonically conjugate variables for the Korteweg-de Vries equation and the Toda lattice with periodic boundary conditions." Progress of Theoretical Physics 55 (1976): 438-56.

[6] Garnett, J. Applications of Harmonic Measure. University of Arkansas Lecture Notes in Mathematics, 8. New York: Wiley, 1986.

[7] Garnett, J., and E. Trubowitz. "Gaps and bands of one dimensional periodic Schrödinger operators." Commentarii Mathematici Helvetici 59 (1984): 258-312.

[8] Grébert, B., T. Kappeler, and J. Pöschel. "Normal form theory for the NLS equation: A preliminary report." (2003): preprint.

[9] Henrici, A., and T. Kappeler. "Global Birkhoff coordinates for the periodic Toda lattice." (2008): preprint arXiv:0802.4252v1.

[10] Henrici, A., and T. Kappeler. "Birkhoff normal form for the periodic Toda lattice." To appear in Contemporary Mathematics, and (2006): preprint arXiv:nlin/0609045v1.

[11] Kappeler, T., and M. Makarov. “On Birkhoff coordinates for KdV." Annales Henri Poincaré 2 (2001): 807-56.

[12] Kappeler, T., and J. Pöschel. $K d V \& K A M$. Ergebnisse der Mathematik, 3. Folge, 45. Berlin: Springer, 2003.

[13] Kappeler, T., and P. Topalov. "Global well-posedness of $\mathrm{KdV}$ in $H^{-1}(\mathbb{T}, \mathbb{R})$. . Duke Mathematical Journal 135, no. 2 (2006): 327-60.

[14] Marchenko, V. A., and I. V. Ostrovskii, A characterization of the spectrum of Hill's operator, Math. SSSR-Sbornik 97 (1975), 493-554.

[15] McKean, H. P., and K. L. Vaninsky. "Action-angle variables for the cubic Schroedinger equation." Communications on Pure and Applied Mathematics 50 (1997): 489-562.

[16] van Moerbeke, P. "The spectrum of Jacobi matrices." Inventiones Mathematicae 37 (1976): 45-81.

[17] Teschl, G. Jacobi Operators and Completely Integrable Nonlinear Lattices. Mathematical Surveys and Monographs, 72. Providence, RI: American Mathematical Society, 2000.

[18] Toda, M. Theory of Nonlinear Lattices, 2nd enl. ed. Springer Series in Solid-State Sciences, 20. Berlin: Springer, 1989. 